\title{
Assessment of off-axis and in-line electron holography for measurement of potential variations in $\mathrm{Cu}(\mathrm{In}, \mathrm{Ga}) \mathrm{Se}_{2}$ thin-film solar cells
}

\author{
Debora Keller ${ }^{1,2^{*}}$, Stephan Buecheler ${ }^{1}$, Patrick Reinhard ${ }^{1}$, Fabian Pianezzi ${ }^{1}$, Etienne Snoeck ${ }^{3}$, Christophe Gatel ${ }^{3}$, \\ Marta D. Rossell ${ }^{2}$, Rolf Erni ${ }^{2}$ and Ayodhya N. Tiwari ${ }^{1}$
}

\begin{abstract}
Electron holography is employed to study variations of the electrostatic crystal potential in $\mathrm{Cu}(\mathrm{In}, \mathrm{Ga}) \mathrm{Se}_{2}(\mathrm{CIGS})$ thin-film solar cells at different length scales: Long-range potential variations across the layer structure of the solar cell as well as inhomogeneities within the layers are analyzed by off-axis holography. In-line holography is applied to examine the local potential variation across a CIGS grain boundary. The phase reconstruction from a focal series is performed by a modified transport of intensity equation (TIE) which is optimized to reduce common artifacts. For comparison, three different microscopes of different optical configurations were used for in-line holography. Based on the results, the impact of the used microscope as well as further acquisition parameters on the in-line holography measurement is assessed. The measured potential variations are discussed considering the effect of different possible sources that may cause potential fluctuations. It is found that most of the variations are best explained by mean inner potential fluctuations rather than by inhomogeneities of the electronic properties. Finally, the present resolution limit of both methods is discussed regarding the feasibility of future electronic characterization of CIGS by holography.
\end{abstract}

Keywords: In-line, Off-axis, Holography, Transport of intensity equation (TIE), Mean inner potential (MIP), Cu(In,Ga) Se2, Chalcopyrite, Solar cell

\section{Background}

Thin-film solar cells based on $\mathrm{Cu}(\mathrm{In}, \mathrm{Ga}) \mathrm{Se}_{2}$ (CIGS) absorbers have demonstrated conversion efficiencies of up to $21.7 \%$ [1]. But despite the high efficiencies, many loss mechanisms are not yet clearly understood. The absorber layer and the interfacial areas in addition build highly complex structures exhibiting nanoscale gradients and fluctuations in electronic, structural, and compositional properties.

Inhomogeneities in the electronic properties of CIGS can be divided into two groups [2,3]. Firstly, stress and

\footnotetext{
${ }^{*}$ Correspondence: debora.keller@empa.ch

${ }^{2}$ Electron Microscopy Center, Empa - Swiss Federal Laboratories for Materials Science and Technology, Ueberlandstrasse 129,

8600 Duebendorf, Switzerland

Full list of author information is available at the end of the article
}

compositional gradients induce spatially independent fluctuations of the conduction and valence band edges which result in local band gap variations. Whereas the $[\mathrm{Ga}] /([\mathrm{Ga}]+[\mathrm{In}])$ ratio (GGI) particularly impacts the conduction band minimum, the $[\mathrm{Cu}] /([\mathrm{Ga}]+[\mathrm{In}])$ ratio (CGI) influences the valence band maximum. Thus, gradients of the GGI and CGI cause variations of the band gap energy in CIGS [4, 5]. Secondly, doping atoms, impurities, and charge accumulations at microstructural defects cause parallel fluctuations of the valence and conduction band edges [6]. As the intrinsic doping in CIGS is linked to the $\mathrm{Cu}$ concentration, the CGI plays a major role regarding potential fluctuations of the second type [5]. The p-type CIGS absorbers used in record efficiency solar cells are in general grown at sub-stoichiometric $\mathrm{Cu}$ concentrations $(\mathrm{CGI}<1)$ because they facilitate the formation of high-quality pn-junctions. However,

\section{黑 Springer}


Cu-deficient CIGS composites show strong compensation, i.e., the net charge introduced by ionized, acceptorlike $\mathrm{Cu}$ vacancies $\left(\mathrm{V}_{\mathrm{Cu}}^{-}\right)$is compensated by the formation of donor-like $\mathrm{In}_{\mathrm{Cu}}^{2+}$ defects. Hence, it is expected that such defects provoke inhomogeneous charge distributions and may cause potential fluctuations of up to $150 \mathrm{mV}$ in $\mathrm{Cu}$-poor absorbers [7]. Additionally, to the potential fluctuations in the bulk, considerable potential variations are expected at grain boundaries in the polycrystalline CIGS. It is known that substantial changes in composition occur at the grain boundaries. Especially, significant variations of the CGI are usually observed at grain boundaries, but also variations in the GGI as well as the presence of impurities (e.g., Na, K, and O, e.g., [8, 9]). Therefore, it is expected, that the effect of grain boundaries is of particular importance when potential variations in CIGS are considered. It is therefore supposed that the reachable efficiency limit of the solar cell is closely related to the presence of potential variations in CIGS which adversely affect the carrier transport and restrict the splitting of the quasi-Fermi levels [2, 3, 7]. However, a tool that allows for characterizing those fluctuations is still needed. It should then open new possibilities to study their impact on the solar cells properties and to further improve their efficiencies in a targeted way.

Electron holography $(\mathrm{EH})$ provides a unique and powerful tool to access information about the electronic properties in nanometer scale. In contrast to conventional transmission electron microscopy (TEM) which only reveals amplitude information on the aberrated exit-plane wave (EPW) transferred to the image plane, holography allows for retrieving the amplitude and the phase of the unaffected EPW [10]. The phase shift, which the incident electron wave experiences while interacting with the specimen and the stray fields surrounding it, contains information about the variation of the composition-dependent crystal potential, about the electrostatic fields in and around the specimen, about the electronic and magnetic properties and about strain. Two of the most common holographic techniques are off-axis EH and bright field through focus in-line EH. Both of these techniques have widely been used to characterize homogeneous and monocrystalline material systems, such as silicon-based devices (e.g., [11-16]), nitrides (e.g., [17, 18]), GaAs systems (e.g., [19]), single nanoparticles (e.g., [20-22]), or simple interfaces (e.g., [23-26]). However, only few EH studies have been reported on more complex materials, such as the CIGS compound (e.g., [6, 27, $28]$ ). Therefore, we apply both of these techniques in the present work to perform potential measurements on CIGS thin-film solar cells and analyze the possibilities and limitations of the two techniques in particular for the study of electronic properties in CIGS in future $[2,3,7]$.
Off-axis holography provides access to phase information by interfering the EPW with a reference wave which travels through vacuum. Using a positively charged biprism allows for directly acquiring a hologram, based on which the phase is retrievable in a simple and direct Fourier-based reconstruction procedure which is described elsewhere [29]. In-line holography is based on the extraction of the phase of the propagating electron wave deduced from the electron beam intensity measured at different defocus. Two different strategies have been proposed in literature to reconstruct the phase information from a focal series: either the phase information is retrieved by iterative algorithms (e.g., [24]) or by the transport of intensity equation (TIE) (e.g., [30]). In contrast to iterative reconstruction routines which require high computational effort, the TIE allows to calculate the phase variation in a direct approach. This work focuses on an advanced TIE-based reconstruction procedure which is described in detail in the "Methods" section.

In contrast to off-axis holography, in-line holography implies no direct restrictions concerning the region of interest on the specimen or the microscope equipment because neither a biprism nor a vacuum reference beam is needed. However, the phase reconstruction procedure is more complex compared to off-axis holography. This sets indirect restrictions on the microscope in order to avoid artifacts. Since all differences between images of different defocus that are not related to phase changes generate artifacts, an aberration-corrected microscope is advantageous and focus-dependent changes of the magnification and rotation as well as image distortion should be minimized. Furthermore, the field of view within which the TIE reconstruction is valid is related to the coherence length of the electron beam. Therefore, a highly (spatially) coherent electron source is required to allow reliable reconstruction within a few tens of nanometers $[25,31]$. Finally, external fields or the presence of diffraction effects which are largely unavoidable in polycrystalline materials introduce artifacts as well. The latter depends mainly on the material and is especially pronounced in in-line holography, as the diffraction effects strongly vary with defocus.

Within the phase object approximation, the phase shift of the electron wave, measured by $E H$, is proportional to the total potential variation seen by the electron in the crystal. For crystals the phase object approximation is not generally correct, since it neglects diffraction contrast effects. But if the relative orientation of the crystal with respect to the electron beam is kept constant and low-index zone axes are avoided, then these effects may in many cases be neglected. According to Eq. (1), the total electrostatic potential variation $\left(V_{\text {total }}\right)$ is then obtained 
based on the phase variation, where $\phi$ is the phase shift of the EPW, $t$ is the thickness of the TEM specimen, and $C_{\mathrm{E}}$ is an interaction constant which depends on the acceleration voltage of the microscope $\left(C_{\mathrm{E}, 200 \mathrm{kV}}=7.27 \mathrm{mradV}^{-1}\right.$ $\left.\mathrm{nm}^{-1} ; C_{\mathrm{E}, 300 \mathrm{kV}}=6.52 \mathrm{mradV}^{-1} \mathrm{~nm}^{-1}\right)$.

$$
V_{\text {total }}=\frac{\phi}{C_{\mathrm{E}} t} \text {. }
$$

Besides the potential fluctuations due to varying electronic properties, further inhomogeneities contribute to the total potential variation that is encountered by the electron beam: In a non-magnetic material $V_{\text {total }}$ may be divided into four different contributions according to Eq. (2): (1) the mean inner potential (MIP), (2) the potential variation due to local redistribution of free charge carriers $\left(V_{\mathrm{E}}\right),(3)$ the potential contribution due to crystal lattice distortions $\left(V_{\text {Dist }}\right)$, and finally, (4) the potential variation caused by electrostatic fields in and around the specimen ( $\left.V_{\text {Fields }}\right)$, e.g., due to charging [32]. Thus, the separation of contributions is generally not trivial.

$$
V_{\text {total }}=M I P+V_{\mathrm{E}}+V_{\text {Dist }}+V_{\text {Fields }} .
$$

The MIP is defined as the average electrostatic potential in a crystalline material which can be estimated by Eq.
(3), where $\Omega$ is the volume of the unit cell, $n_{\mathrm{j}}$ is the number of atoms of species $\mathrm{j}$ per unit cell, and $f_{\mathrm{j}}(\mathbf{q}=0)$ is the atomic elastic scattering amplitude for electrons in forward direction, with $\mathbf{q}$ being a spatial frequency vector [33].

$$
M I P=\frac{h^{2}}{2 \pi m e \Omega} \sum_{j} n_{j} f_{j}(\mathbf{q}=0) .
$$

Literature values of scattering factors for electrons in forward direction of the relevant elements are summarized in Table 1 which gives the reported and estimated scattering factors for the different atoms and the corresponding ions (e.g., [33-35]). Further, the MIP values for the relevant compounds are calculated according to Eq. (3) and are also shown in Table 1.

\section{Methods}

\section{Experimental details}

Sample preparation

Two CIGS thin-film solar cell devices were produced on flexible polyimide foils according to the recipe described by Chirilă et al. [36, 37], including a post-deposition treatment of the CIGS absorber layer with $\mathrm{Na}$ and $\mathrm{K}$. The

\begin{tabular}{|c|c|c|c|c|}
\hline \multirow[t]{2}{*}{ Element } & \multicolumn{2}{|c|}{ Atomic scattering factor (nm) } & \multicolumn{2}{|c|}{ lonic scattering factor (nm) } \\
\hline & Ref. [34] & Ref. [33] & Ref. [35] & Ref. [33] \\
\hline $\mathrm{Cu} / \mathrm{Cu}^{+}$ & 0.55 & 0.63 & 0.33 & $0.45^{* *}$ \\
\hline $\ln / \ln ^{3+}$ & 1.03 & 1.06 & 0.46 & $0.29 *$ \\
\hline $\mathrm{Ga} / \mathrm{Ga}^{3+}$ & 0.70 & 0.71 & 0.22 & $0.18^{*}$ \\
\hline $\mathrm{Se} / \mathrm{Se}^{2-}$ & 0.72 & 0.73 & $0.95^{*}$ & $1.02 *$ \\
\hline $\mathrm{Cd} / \mathrm{Cd}^{2+}$ & 0.92 & 0.92 & 0.51 & 0.51 \\
\hline$S / S^{2-}$ & 0.52 & 0.52 & $0.65^{*}$ & $0.71^{*}$ \\
\hline $\mathrm{Zn} / \mathrm{Zn}^{2+}$ & 0.60 & 0.60 & 0.26 & 0.26 \\
\hline $\mathrm{O} / \mathrm{O}^{2-}$ & 0.20 & 0.20 & 0.35 & 0.41 \\
\hline \multirow[t]{2}{*}{ Compound } & \multicolumn{2}{|l|}{$\mathrm{MIP}[\mathrm{V}]$} & \multicolumn{2}{|l|}{$\mathrm{MIP}[\mathrm{V}]$} \\
\hline & Ref. [34] & Ref. [33] & Ref. [35] & Ref. [33] \\
\hline CulnSe $_{2}$ & 14.8 & 15.5 & 13.3 & 13.7 \\
\hline $\mathrm{CuGaSe}_{2}$ & 14.9 & 15.5 & 13.6 & 14.8 \\
\hline $\mathrm{ClGS}_{\mathrm{GGl}}=0.36$ & 14.8 & 15.5 & 13.4 & 14.0 \\
\hline $\mathrm{CdS}$ & 13.5 & 13.6 & 11.0 & 11.6 \\
\hline $\mathrm{ZnO}$ & 16.1 & 16.2 & 12.2 & 13.5 \\
\hline
\end{tabular}

Table 1 Scattering factors for electrons in forward direction for relevant atoms and corresponding ions in CIGS solar cells according to Weickenmeier et al., Rez et al. and Peng et al. [33-35]

Furthermore, the MIP values for some relevant compounds are listed below under the assumption of either fully covalent or fully ionic bonds. The scattering factors marked by * in the table show estimated values, as these values were not recorded in the corresponding literature source. The values of the scattering factors $f\left(\mathrm{e}^{2-}\right.$ ) and $f\left(\mathrm{~S}^{2-}\right)$ were extrapolated from the reported value $f\left(\mathrm{O}^{2-}\right)$ assuming that the scattering factors increase linearly by a factor $I_{\mathrm{O}}^{2-}$ with increasing period number of the considered group in the periodic table. The factor $I_{\mathrm{O}}^{2-}$ was calculated as $I_{\mathrm{O}}^{2-}=I_{\mathrm{O}}^{2-}\left(\mathrm{I}_{\mathrm{F}}-/ I_{\mathrm{F}}\right)$ whereas the linear increase factor $I_{O}$ was calculated based on the values $f(\mathrm{O}), f(\mathrm{~S})$, and $f(\mathrm{Se})$. The linear increase factor $I_{\mathrm{F}}$ was calculated based on the values $f(\mathrm{~F}), f(\mathrm{Cl}), f(\mathrm{Br})$, and $f(\mathrm{I})$ and the linear increase factor $I_{\mathrm{F}}-$ was calculated based on the values $f(\mathrm{~F}), f(\mathrm{Cl}), f(\mathrm{Br})$, and $f(\mathrm{I})$. The values of the scattering factors $f\left(\mathrm{Ga}^{3+}\right)$ and $f\left(\mathrm{In}^{3+}\right)$ were extrapolated likewise based on the reported values $\left.f(\mathrm{Al})^{3+}\right), f(\mathrm{Al}), f(\mathrm{Ga})$, $f(\mathrm{In}), f(\mathrm{Mg}), f\left(\mathrm{Mg}^{2+}\right), f(\mathrm{Ca}), f\left(\mathrm{Ca}^{2+}\right), f(\mathrm{Sr})$, and $f\left(\mathrm{Sr}^{2+}\right)$. The value $f\left(\mathrm{Cu}^{+}\right)$(marked by $\left.\left.{ }^{* *}\right)\right)$ is approximated as the average of $f\left(\mathrm{Cu}{ }^{2+}\right)$ and $f(\mathrm{Cu})$ 
thin-film solar cell stack consists of the following layers: polyimide (PI) foil ( $\sim 25 \mu \mathrm{m}$, substrate), Mo $(\sim 600$ $\mathrm{nm}$, back contact), co-evaporated CIGS $(\sim 2 \mu \mathrm{m}, \mathrm{p}$-type absorber layer), $\operatorname{CdS}(\sim 20 \mathrm{~nm}, \mathrm{n}$-type buffer layer), $\mathrm{i}-\mathrm{ZnO}$ ( $\sim 50-60 \mathrm{~nm}$, front contact), and $\mathrm{ZnO}: \mathrm{Al}(\sim 200$ $\mathrm{nm}$, front contact), $\mathrm{Ni} / \mathrm{Al} / \mathrm{Ni}$ grid $(\sim 4 \mu \mathrm{m}$, the grid is not totally covering the surface and therefore not visible in TEM images), $\mathrm{MgF}_{2}(\sim 100 \mathrm{~nm}$, anti-reflection coating). A sketch of the solar cell set-up superimposed to a low magnification scanning (S-)TEM image is shown in Fig. 1.

The two solar cells differ in the composition of their absorber layer. The first absorber layer was co-evaporated with a standard CIGS composition (low Cu content) and the second with high $\mathrm{Cu}$ content. In Table 2, the average composition of the absorber layer as measured by X-ray fluorescence measurements (XRF) and the characteristic properties of the two solar cells are summarized. As the CIGS growth receipt was modified in order to change the $\mathrm{Cu}$ content, the differences in efficiency of those two cells are not attributed to the difference in $\mathrm{Cu}$ content only, but also to other parameters such as the $\mathrm{Ga} / \mathrm{In}$ grading and the band gap energy at the interface.

Two different kinds of TEM specimens were prepared from both solar cells: Firstly, cross-sectional stacks were conventionally polished and then $\mathrm{Ar}^{+}$-ion milled with a Bal-Tec RES 101 and a Fischione TEM ion mill 1050. This

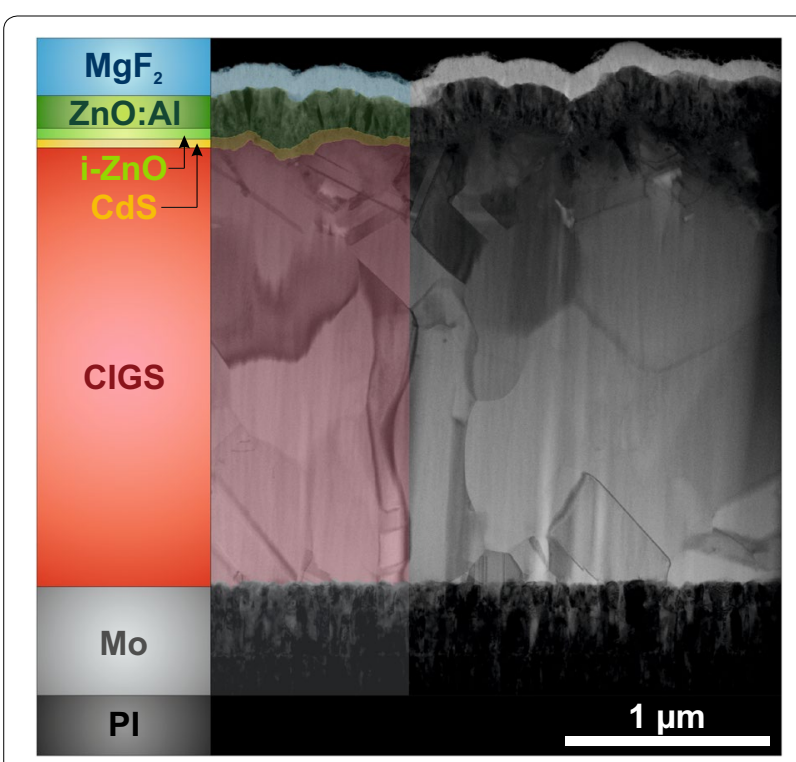

Fig. 1 CIGS solar cell. Set-up of a standard CIGS solar cell on flexible PI. The layer stack consists of the following layers from bottom to top: Pl substrate $(\sim 25 \mu \mathrm{m})$, Mo back contact $(\sim 600 \mathrm{~nm})$, co-evaporated CIGS absorber layer ( 2 $\mu \mathrm{m})$, CdS buffer layer ( $\sim 2 \mathrm{~nm}), \mathrm{i}-\mathrm{ZnO}(\sim 50-60$ $\mathrm{nm})$, and $\mathrm{ZnO}: \mathrm{Al}(\sim 200 \mathrm{~nm})$ bilayer front contact, $\mathrm{MgF}_{2}$ anti-reflection coating $(\sim 100 \mathrm{~nm})$
Table 2 Two CIGS thin-film solar cells were produced having a standard (low Cu content) CIGS absorber layer or a CIGS with high Cu content

\begin{tabular}{lll}
\hline & $\begin{array}{l}\text { Standard CIGS solar cell } \\
\text { (low Cu content) }\end{array}$ & $\begin{array}{l}\text { Solar cell with CIGS } \\
\text { of high Cu content }\end{array}$ \\
\hline GGI (CIGS) [-] & 0.36 & 0.37 \\
CGI (CIGS) [-] & 0.79 & 0.99 \\
$V_{O C}[\mathrm{~V}]$ & 0.70 & 0.65 \\
$J_{S C}\left[\mathrm{mACm}^{-2}\right]$ & 34.6 & 34.0 \\
FF $[\%]$ & 76.7 & 71.1 \\
$\eta[\%]$ & 18.6 & 15.7 \\
\hline
\end{tabular}

Their characteristic properties are summarized below, whereas $V_{O C}$ is the opencircuit voltage, $J_{\mathrm{SC}}$ is the short-circuit current density, $F F$ is the fill factor, and $\eta$ the efficiency of the completed solar cell

preparation procedure applies to the results obtained at the CIGS grain boundary. Secondly, TEM lamellae were prepared by in-situ lift-out, followed by thinning with a focused $\mathrm{Ga}^{+}$-ion beam (FIB). First a $10 \mathrm{~nm}$ Pt protection layer was sputtered on top of the solar cell by a Leica EM ACE600 coater. Then an additional Pt/C layer was deposited by FIB ( $\mathrm{Ga}^{+}$-ion beam). A FEI Helios NanoLab 600i DualBeam was used with a milling voltage of $30 \mathrm{kV} \mathrm{Ga}$ -ion milling, followed by 5 and $2 \mathrm{kV}$ milling in the final stages. FIB lamellae were used for off-axis holography experiments.

\section{Microscopes and experimental set-up}

In-line holography was performed on three different microscopes: Firstly a Jeol 2200FS TEM/STEM was used equipped with a Schottky field emitter and operated at $200 \mathrm{kV}$. An (in-column) energy filter with an energy slit of $10 \mathrm{eV}$ prevented inelastically scattered electrons from contributing to the image. Further, an objective aperture was inserted to reduce diffraction effects. Images were acquired for $20 \mathrm{~s}$ using a charge-coupled device (CCD) camera with $2048 \times 2048$ pixels. The noise level in the focal series was in a range of $3.5 \pm 0.3 \%$.

Secondly, an in-line $\mathrm{EH}$ experiment was performed on a Jeol ARM 200F equipped with a cold FEG (200 $\mathrm{kV}$ ) with $\mathrm{C}_{\mathrm{s}}$-correctors in the imaging and illumination part (CETCOR, CESCOR from CEOS GmbH). A small objective aperture was inserted to reduce diffraction effects. The acquisition time was $5 \mathrm{~s}$ and a CCD camera with $2048 \times 2048$ pixels was used. The images contained roughly $2.1 \pm 0.2 \%$ noise.

And thirdly, the Hitachi I2TEM microscope (HF3300C) was used, equipped with a cold FEG operated at $300 \mathrm{kV}$ fitted with an advanced imaging $\mathrm{C}_{\mathrm{S}}$-corrector (BCOR, CEOS $\mathrm{GmbH}$ ) which fully corrects for off-axial coma, and 4 rotatable electrostatic biprisms. Inelastically scattered electrons were filtered by the biprisms 
and hologram images were acquired for $5 \mathrm{~s}$ on a CCD camera with $4096 \times 4096$ pixels. Subsequently, the focal stack was built by extracting the center band, i.e., the amplitude information of the hologram, for each image. The noise level of these images was in the range of 1.2 $\pm 0.03 \%$.

Off-axis holography was performed on the Hitachi I2TEM. The biprisms were aligned perpendicular to the elliptical illumination. Two biprisms behind the sample were employed. A voltage of $40 \mathrm{~V}$ was applied to the first biprism and $193 \mathrm{~V}$ to the second biprism for the results presented in Figs. 4 and 6, respectively, $226 \mathrm{~V}$ to the second biprism for the results presented in Fig. 9. The holograms which were obtained with a biprism voltage (lower biprism) of $193 \mathrm{~V}$, reveal a fringe contrast of $13.8 \%$, a fringe spacing of $1.17 \mathrm{~nm}$, and a hologram width of 450 $\mathrm{nm}$. In the case of $226 \mathrm{~V}$ applied voltage on the second biprism, the hologram width remains $450 \mathrm{~nm}$, but the fringe spacing decreases to $0.95 \mathrm{~nm}$ while the fringe contrast is reduced to $7.7 \%$. Again, the acquisition time was $5 \mathrm{~s}$ using a CCD with $4096 \times 4096$ pixels.

\section{Modified TIE reconstruction procedure Basic TIE}

The TIE was first described by Teague as an intensity conservation equation which was later on solved within various approaches by Paganin and Nugent [30, 38]. A detailed derivation of the TIE (Eq. 4) is given by De Graef [39]. The TIE relates the intensity derivative of several images at different defoci to the lateral phase variation (Eq. 4), where $(\phi(x, y, z))$ is the lateral phase variation, $\left(\frac{\delta}{\delta z} I_{0}\right)$ is the intensity derivative at $\mathrm{z}=0, I_{0}$ is the intensity at $\mathrm{z}=0, \lambda$ is the electron wave length, and $\nabla_{\mathrm{x}, \mathrm{y}}$ the two-dimensional gradient operator in $x$ and $y$, resp. $\nabla_{x, y}^{-2}$ its inverse.

$$
\phi(x, y, z)=-\frac{2 \pi}{\lambda}\left(\frac{1}{I_{0}} \nabla_{\mathrm{x}, \mathrm{y}} \nabla_{\mathrm{x}, \mathrm{y}}^{-2} \frac{\delta}{\delta z} I_{0}\right) .
$$

In this work, the simple TIE was implemented as a script in Digital Micrograph. Then the algorithm was further adapted and improved as described in the following. The effect of the different improvement steps is exemplarily shown in Figs. 2 and 3, based on a simulated focal series. Figure 2 shows (a) the amplitude and (b) the original phase image, as well as (c) the overfocus image which is simulated using (a) and (b). Amplitude and phase images were arbitrarily chosen for visualization of the algorithm. In an experimentally acquired focal series it is generally not possible to achieve a perfect alignment of the focal stack prior to the phase reconstruction because the images vary in defocus and are thus not identical. In order to demonstrate the effect of small residual misalignments in a real measurement, all defocus images used in the simulation were subjected to a random shift in respect to each other by $<2 \%$ of the image size. The phase image (shown in Fig. 3a) is retrieved by the simple TIE using three images, i.e., Fig. 2a, c as well as the corresponding underfocus image.

Symmetrization The numerical solution of the TIE includes the application of the fast Fourier Transform (FFT) and its inverse, which implies periodic boundary conditions in the phase image (Eq. (5), where $\mathbf{q}_{\mathrm{i}}$ and $\mathbf{q}_{\mathrm{x}, \mathrm{y}}$ are reciprocal vectors used for normalization). However, this condition is generally violated in real samples. As a consequence, artifacts appear at the edges of the reconstructed phase image and gradients over the whole reconstructed phase are generated. However, as reported by Volkov et al., these artifacts may in many cases be circumvented if the images are mirrored at the $\mathrm{x}$ - and $\mathrm{y}$-axis before applying the TIE. Then, only the gradient of the phase normal to the boundaries must be zero at the boundary but not the absolute values of the phase or amplitude. Therefore, the so-called symmetrization allows for eliminating these types of artifacts for a wide range of objects. The symmetrization

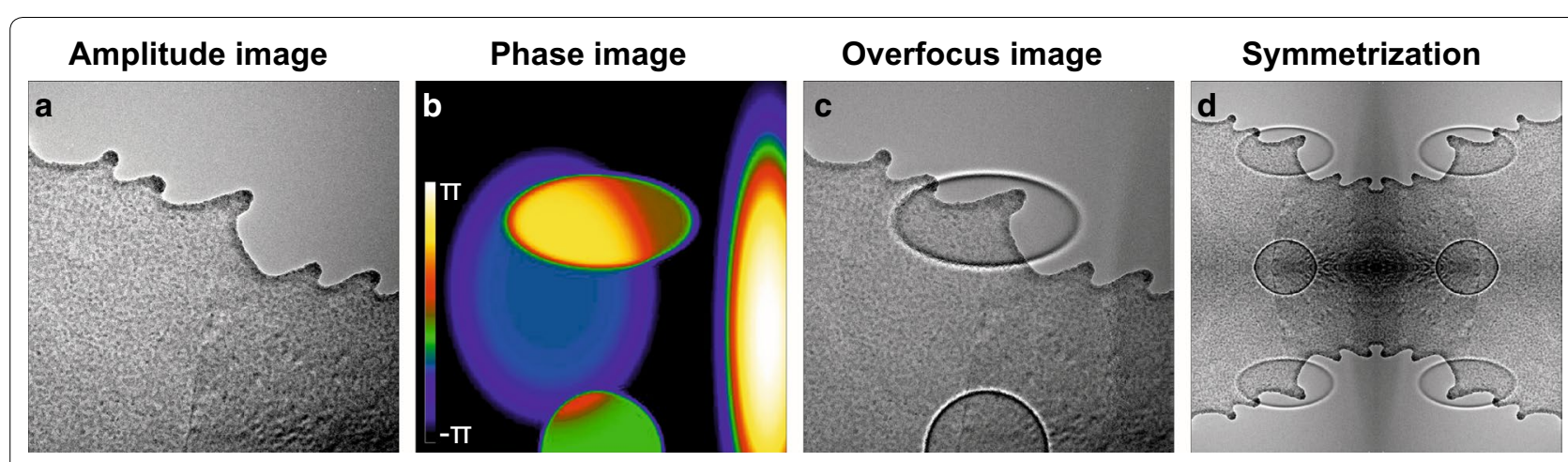

Fig. 2 Test images used for simulations. a Amplitude image and $\mathbf{b}$ phase image used for phase reconstruction simulations. In c a simulated overfocus image is shown and $\mathbf{d}$ depicts an example of the extended overfocus image using symmetrization 


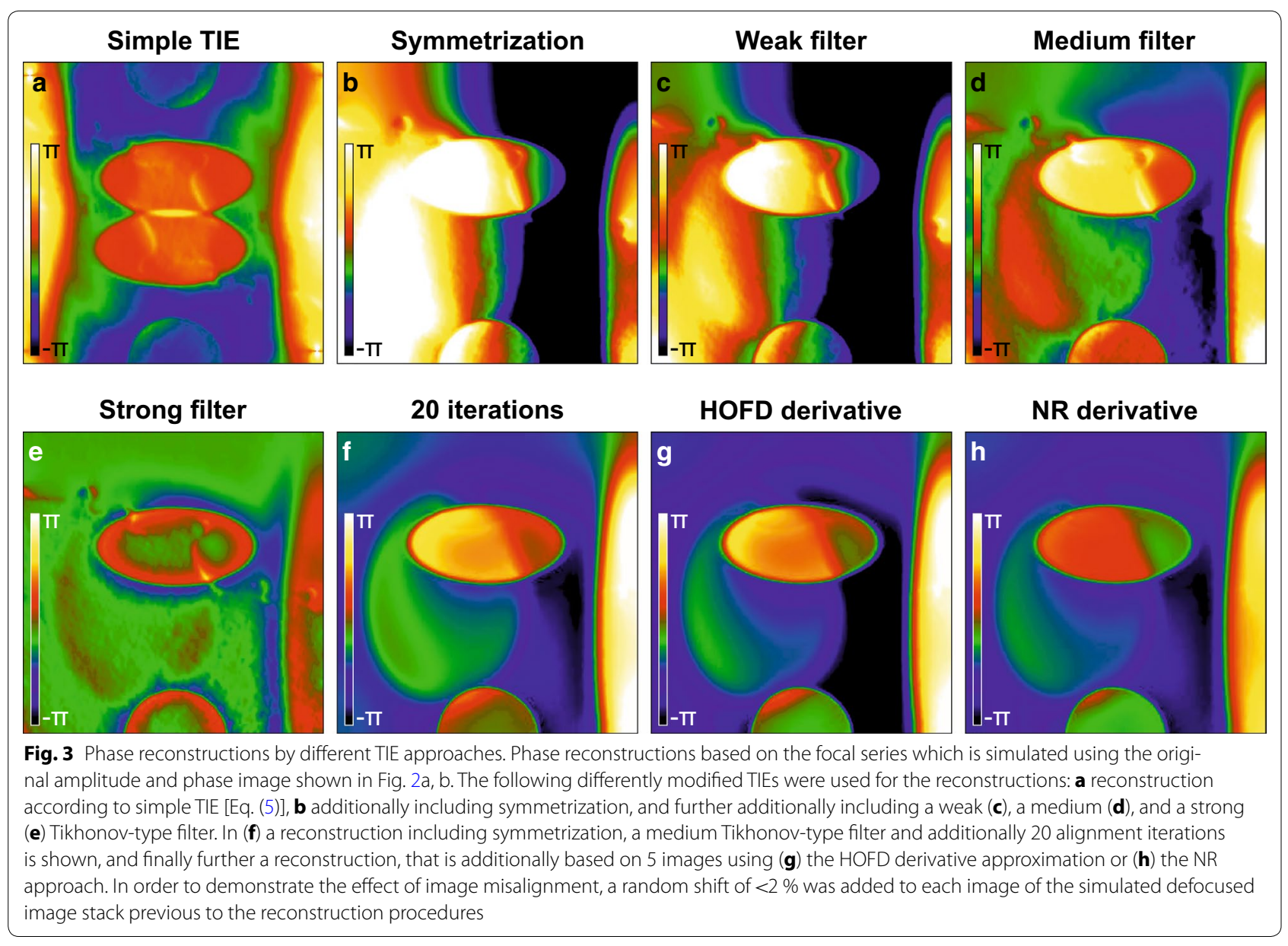

of the simulated overfocus image is exemplarily shown in Fig. 2d [22] and the improvement of the phase image due to the implementation of symmetrization into the simple TIE algorithm is demonstrated in Fig. 3b.

$$
\begin{aligned}
& \phi(x, y, z) \\
& =-\frac{2 \pi}{\lambda} F^{-1} \sum_{i=1}^{2}\left(Q F\left(\frac{1}{I_{0}} F^{-1}\left\{Q F\left[\frac{\delta}{\delta z} I_{0}\right]\right\}\right)\right) \\
& \text { with } \\
& Q=\frac{2 \pi \mathbf{q}_{\mathrm{i}}}{\mathbf{q}_{\mathrm{x}, \mathrm{y}}^{2}}
\end{aligned}
$$

Low-frequency filtering Another limiting characteristic of the TIE is the $1 / \mathbf{q}^{2}$-low-pass filter effectuated by the gradient operator $\nabla_{x, y}^{-2}$, which results in an enhancement of low-frequency noise in the reconstructed phase image and blurring of sharp edges. In order to overcome these issues, different approaches have been reported in literature. The subtraction of a linear background $[23,26]$ and the selection of small sub-regions $[26,40]$ were used to subtract low-frequency noise in phase reconstruction procedures where vacuum regions were present in the images. Ishizuka and Allman proposed to suppress low frequencies with a Tikhonov-type filter which is also applicable if no vacuum region is present in the selected region of interest [41]. This filter is applied to the gradient operator, according to Eq. (6), where $\mathbf{q}_{\mathbf{c}}$ is the cut-off frequency in reciprocal space. The reciprocal length $\mathbf{q}_{\mathrm{c}}$ defines the strength of the filter and needs to be optimized in order to suppress noise while still maintaining the relevant phase modulations.

$$
T(\mathbf{q})=\frac{\mathbf{q}^{2}}{\mathbf{q}^{2}+\mathbf{q}_{\mathrm{c}}^{2}} .
$$

Examples of phase images which are obtained by applying three different Tikhonov-type filters are presented in Fig. 3c-e. As demonstrated, the optimum choice of the filter strength is crucial. While a too weak filter (Fig. 3c) does not reduce the background gradient sufficiently, a too strong filter (Fig. 3e) causes essential frequencies to be lost. In the latter case, the magnitude of phase variations is damped and the absolute values are therefore not reliable anymore. 
Iterative alignment Small misalignments of the focal series may already cause severe artifacts in the phase image as demonstrated by Barty et al. [42]. Generally, residual misalignment of the focal series cannot be avoided because the difference between the images due to the varying defocus hampers a perfect alignment. Therefore, an iterative in-situ alignment was further implemented into the TIE routine. After the first phase image has been reconstructed, the procedure allows simulating the defocus images based on the reconstructed phase and the in-focus image. Then, the experimental defocus images are aligned according to the simulated focal series and another slightly improved phase image is calculated. As shown in Fig. 3f, the obtained phase image in our example is clearly improved after using 20 alignment iterations.

Intensity derivative approximation The phase reconstruction using the TIE is based on the intensity derivative and thus, inaccuracies in the estimation of the intensity derivative crucially affect the phase retrieval. In the basic approach which uses three images the intensity derivative is approximated by two defocus images, assuming linearity according to Eq. (7). Hence, the error due to non-linearity of the derivative increases exponentially with increasing defocus of the two images $\left(\sim \delta z^{2}\right)$ and affects mainly the magnitude of the measured phase variation. Further, also the spatial resolution of the retrieved phase image deteriorates if larger defocus values are used [43]. In contrast, if small defoci are chosen, the noise in the defocus images causes distortions of higher significance and reduces the phase resolution. Therefore, the optimal defocus is a trade-off between reducing the effect of noise and reducing inaccuracies due to the linear derivative approximation [41].

$$
\frac{\delta}{\delta z} I_{0}=\frac{I(x, y, z+\Delta z)-I(x, y, z-\Delta z)}{2 \Delta z} .
$$

In order to improve the estimation of the derivative and therefore allowing for the use of larger defoci, more advanced approaches have been proposed in literature. The higher-order finite difference (HOFD) method estimates a non-linear derivative based on $2 n+1$ images of different defocus, according to Eq. (8), where $I(x, y, z+i \Delta z)$ is a set of defocus images with $i=-n, \ldots, 0, \ldots, n$ and where $a_{\mathrm{i}, \text { HOFD }}$ are the image weight coefficients $[41,44]$. Soto and Acosta proposed another set of image weight coefficients $a_{\mathrm{i}, \mathrm{NR}}$ which focuses stronger on noise reduction (NR) [45].

$$
\begin{aligned}
& \frac{\delta}{\delta z} I_{0}=\sum_{i=-n}^{n} \frac{a_{\mathrm{i}} I(x, y, z+i \Delta z)}{\Delta z} \\
& \text { with } \\
& a_{\mathrm{i}, \mathrm{HOFD}}=\frac{(-1)^{i+1}(n !)^{2}}{i(n+1) !(n-1) !} \\
& \text { or } \\
& a_{\mathrm{i}, \mathrm{NR}}=\frac{3 i}{n(n+1)(2 n+1)} .
\end{aligned}
$$

By using these approaches better reconstructions can be achieved, even though the consideration of more images implies additional noise and misalignment in real focal series. However, the optimal number of images and their defocus needs to be evaluated to achieve the most accurate phase image. In Fig. $3 g$, h, a phase reconstruction example of both, the HOFD method and the NR approach are shown, each using five images (an underand overfocus image of twice as large defocus are used additionally). As compared to Fig. 3f, low-frequency artifacts are further suppressed by both approaches (g) and (h). Since no noise is artificially added in the simulated series, the HOFD approach leads to better results than the NR approach. In the performed in-line holography experiments, where real, noisier series are treated, a stack of five images combined with the NR approach turned out to be most suitable.

\section{Results and discussion}

\section{Potential variations across the pn-junction}

Off-axis holograms of the two solar cells were acquired in order to analyze the phase variation across the upper part of the solar cell layer stack, especially the CIGS/CdS interface region. In Fig. 4a, b, a mosaic of four reconstructed phase images across the different layers of the solar cell with the standard CIGS (low $\mathrm{Cu}$ content) absorber layer is presented as an overview. Further, high-angle annular dark-field (HAADF-) and bright-field (BF-) STEM micrographs of the corresponding area are shown in Fig. 4c, d, where the stacking of the different solar cell layers (CIGS, $\mathrm{CdS}, \mathrm{ZnO}, \mathrm{MgF}_{2}$ ) is indicated. To analyze phase shift gradients through and within the different layers of the solar cell, line profiles (Fig. 5a, b) of the phase variation were extracted perpendicular to the layer stack within the boxes $\left(A_{1}, A_{2}, B_{1}, B_{2}\right)$ marked in Fig. $4 a$, b. At the same position, line profiles (Fig. 5a, b) were obtained from the HAADFSTEM images in A and B. The phase images in Fig. 4( $\mathrm{a}_{1}$, $b_{1}$ ) were normalized by setting the phase shift in vacuum to zero, the phase images in Fig. $4\left(a_{2}\right)$ and $\left(b_{2}\right)$ were normalized by matching their overlap region with the phase images in Fig. $4\left(a_{1}\right)$ and $\left(b_{1}\right)$. Nevertheless, a residual 

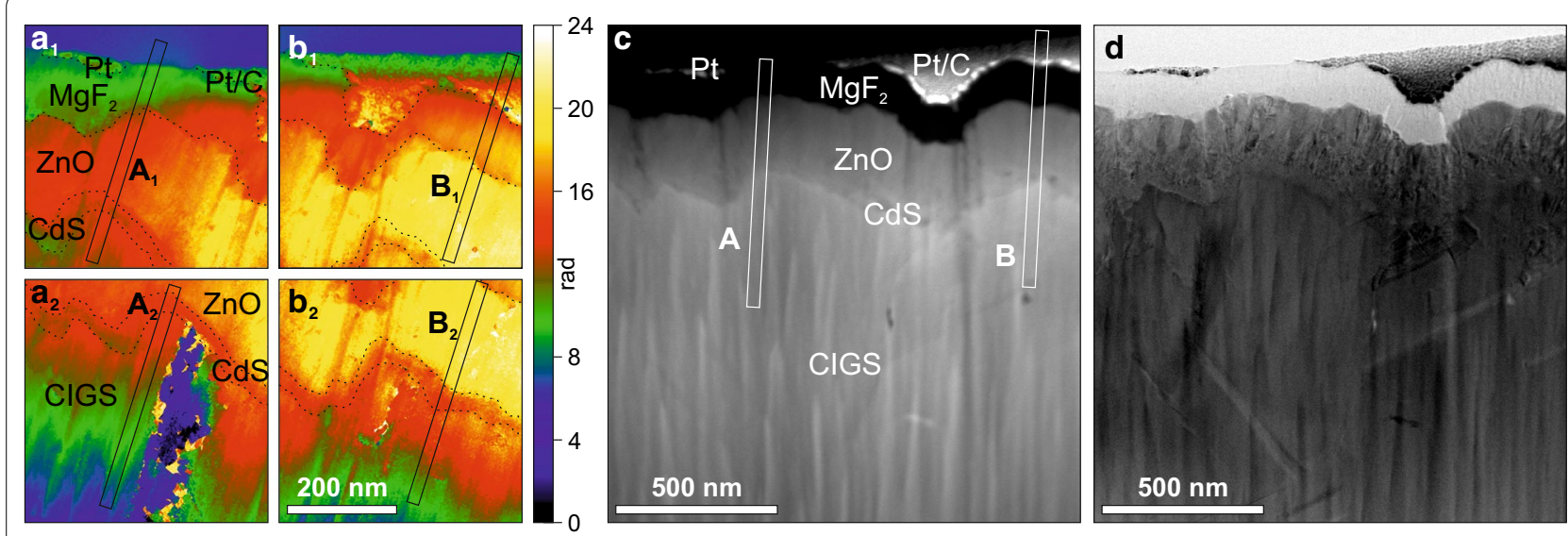

Fig. 4 Off-axis holography on standard CIGS solar cell. a, b Reconstructed phase images from off-axis holograms acquired on a solar cell with standard CIGS (GGI 0.36, CGI 0.79). c HAADF-STEM and d BF-STEM micrographs show the same region of the solar cell. The intensity profiles, that were extracted from the phase images and the HAADF-STEM image within the marked boxes $\left(A_{1}, A_{2}, B_{1}\right.$, and $B_{2}$, resp. A and $\left.B\right)$, are given in Fig. 5a, b

background gradient remained in the reconstructed phase images, which might stem from specimen charging under the electron beam and therefore may affect $V_{\text {Fields. }}$. Thus, the extracted profiles shown in Fig. 5a, b were further corrected by subtracting an additional linear background gradient. This gradient was approximated based on the HAADF intensity such that the long-range thickness variation measured by the phase fits best to the expected, relative long-range thickness variation measured by the HAADF-STEM intensity.

Based on the HAADF-STEM intensities ( $I_{\text {HAADF-STEM }}$ ), the relative thickness variation $\left(t_{\text {HAADF-STEM }}\right.$, Fig. $5 \mathrm{c}, \mathrm{d}$ was estimated according to Eq. (9), where $Z$ is the average atomic number of the compound and $\rho_{\text {at }}$ is the density of atoms per unit volume. The average atomic number of each layer was determined under the assumption that the composition is homogeneous.

$$
t_{\text {HAADF-STEM }} \propto \frac{I_{\text {HAADF-STEM }}}{Z^{2} \rho_{\text {at }}} .
$$

Likewise, based on the phase images the absolute thickness $\left(t_{\text {phase }}\right.$ Fig. 5 c, d) was calculated according to Eq. (1), assuming that the MIP is constant within each layer and corresponds to the values of neutral compounds given in Table 1. Compared to the absolute thickness values of 100-150 nm which were determined by the log-ratio method in Energy Filtered TEM (EFTEM) [46], the thickness measured by the phase is slightly higher. As though residual background gradients may be present, these may affect the precision of the absolute values. In general, by neglecting contributions of $V_{\text {Fields }}$ and $V_{\text {Dist }}$ as well as diffraction effects, any relative disagreement of the two thickness plots $t_{\text {HAADF-STEM }}$ and $t_{\text {phase }}$ of Fig. $5 \mathrm{c}$, d can be assigned to additional potential variations. These may either stem from deviations of the $M I P$ from the expected values, or from local redistributions of free charge carriers $\left(V_{\mathrm{E}}\right)$, given that the used values for the $M I P, Z$, and $\rho_{\text {at }}$ are accurate. Comparing $t_{\text {HAADF-STEM }}$ and $t_{\text {phase }}$ indeed reveals some disagreements:

1. At each interface, spikes that are caused by discontinuities of $Z, \rho_{\text {at }}$ and $M I P$ are observed since the interfaces are neither flat nor perfectly perpendicular to the electron beam. These spikes are considered to be artifacts.

2. Both profiles show a small variation in $t_{\text {phase }}$ in the first $50 \mathrm{~nm}$ of the $\mathrm{ZnO}$ layer above the $\mathrm{CdS} / \mathrm{ZnO}$ surface. In contrast, $t_{\text {HAADF-STEM increases linearly }}$ and thus suggests a linear thickness change of the specimen. Since the $\mathrm{ZnO}$ layer is intrinsically doped within the first $50 \mathrm{~nm}$ and aluminum-doped within the remaining $\mathrm{ZnO}$ layer, it is supposed that the additional potential variation reflects the change of the dopant type. Since the number of free charge carriers is different in the two parts of $\mathrm{ZnO}, V_{\mathrm{E}}$ is affected and may cause a change in $V_{\text {total. }}$. However, the measured potential variation may also be caused by variations of the MIP. In addition to the small amounts of $\mathrm{Al}$ which are added to the upper part of the layer the energy dispersive $x$-ray spectroscopy (EDX) profiles (Fig. 5e, f) also indicate that the $\mathrm{Zn}: \mathrm{O}$ ratio is lower there, compared to the first $50 \mathrm{~nm}$ of the $\mathrm{ZnO}$. Therefore, it is assumed that the compositional changes and possible related changes in the ionicity of the atoms cause a change of the MIP and thus a change in $V_{\text {total }}$ as well.

3. Both thickness profiles, $t_{\text {phase }}$ and $t_{\text {HAADF-STEM }}$, suggest a thickness increase of the specimen in the CdS 

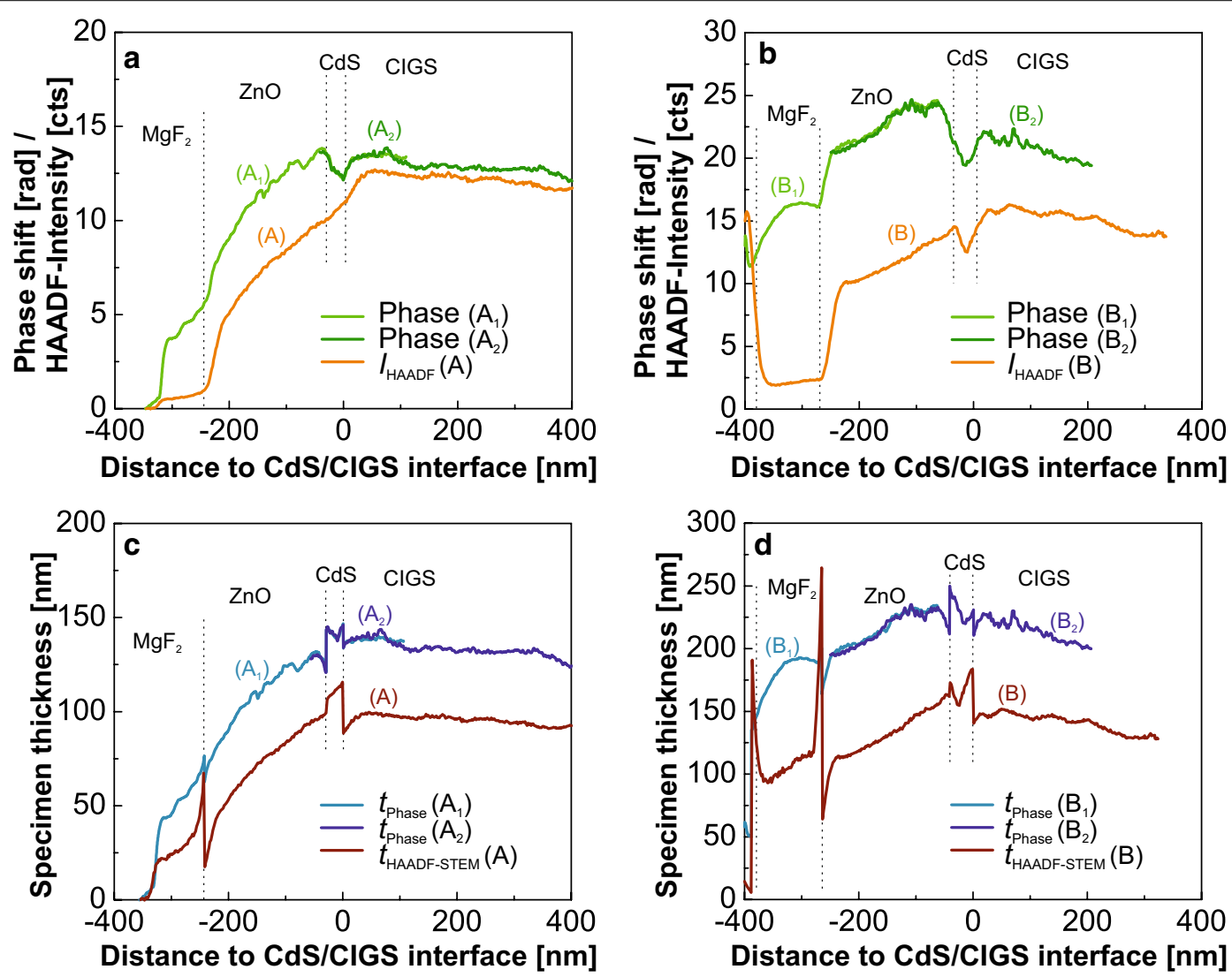

EDX line profiles at region $A$
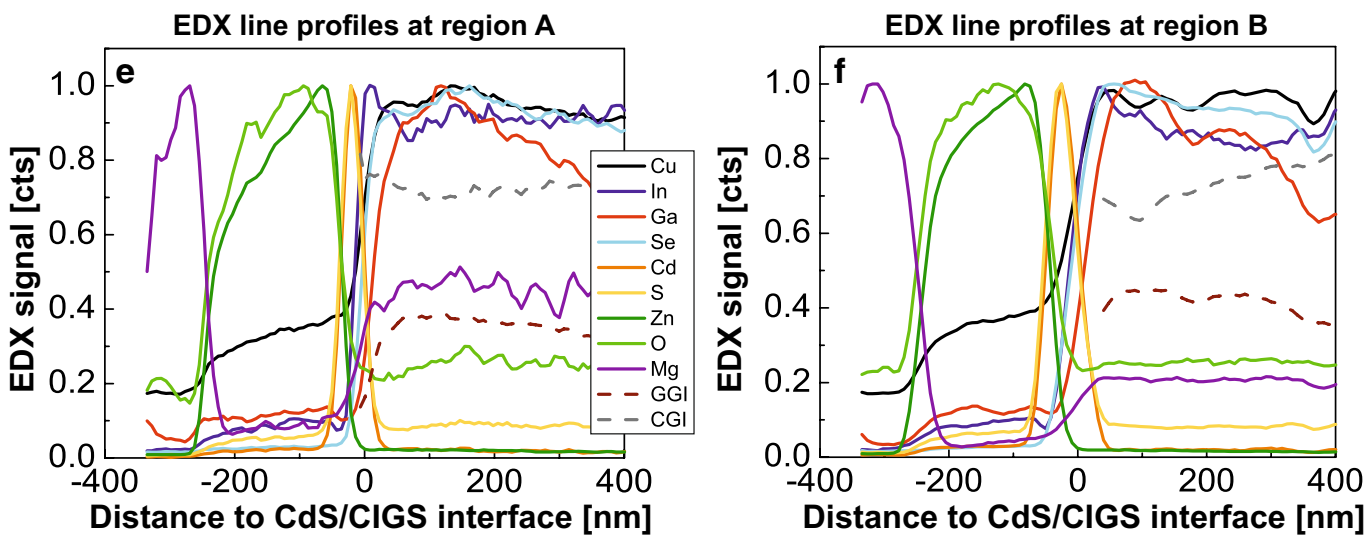

Fig. 5 Phase variations on standard CIGS solar cell. a, b Intensity line profiles showing the phase variations (green line) which are extracted from the regions marked in Fig. 4a, b. Below, the intensity profiles that are extracted from the same regions in the HAADF-STEM image (yellow lines) are shown. In order to correct for residual background gradients in the phase images, an individual linear background was subtracted from each phase variation line profile. The relative thickness variation, measured by HAADF-STEM $\left(I_{\text {HAADF-STEM }}\right)$ and the absolute thickness variation, measured by the phase images ( $t_{\text {phase }}$ ) based on $\mathbf{a}, \mathbf{b}$, are given in $\mathbf{c}$, $\mathbf{d}$. The corresponding EDX profiles are depicted in $\mathbf{e}, \mathbf{f}$ showing relative concentration variations. The copper signal in the $\mathrm{ZnO}$ region originates from stray electrons that impinge on the copper grid. Further a significant amount of $\mathrm{Mg}$ and $\mathrm{O}$ is measured in the CIGS region. These are artifacts due to the overlap of the Se L-lines with the K-lines of Mg and the Cr L-lines (Cr is present in the TEM specimen holder) with the $\mathrm{O}$ K-lines. In addition to the EDX profiles of the present elements, also the relative variation of GGI and CGI is qualitatively shown in the graph 
layer. However, since the specimen is prepared by FIB where $\mathrm{CdS}$ is milled very fast compared to $\mathrm{ZnO}$, the $\mathrm{CdS}$ layer is not expected to be thicker than the $\mathrm{ZnO}$ layer. Since the EDX line profiles (Fig. 5e, f) show rough $\mathrm{ZnO} / \mathrm{CdS}$ - and $\mathrm{CdS} / \mathrm{CIGS}$-interfaces, the apparent thickness increase is better explained by the overlap of $\mathrm{ZnO}$ and CIGS with CdS. Assuming pure $\mathrm{CdS}$, whereas in reality also CIGS or $\mathrm{ZnO}$ is present, leads to an overestimation of the $\mathrm{CdS}$ thickness. Considering $t_{\text {phase, which is based on }}$ the estimation of the MIP, the overlap of $\mathrm{CdS}$ with $\mathrm{ZnO}$ would lead to the larger error than the overlap with CIGS (as $M I P_{\mathrm{CdS}}<M I P_{\mathrm{CIGS}}<M I P_{\mathrm{ZnO}}$ ).

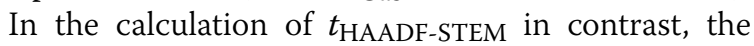
overlap of $\mathrm{CdS}$ with CIGS would lead to a larger error than with $\mathrm{ZnO}$, if pure $\mathrm{CdS}$ is assumed $\left(Z^{2} \rho_{\mathrm{CdS}}<Z^{2} \rho_{\mathrm{ZnO}}<Z^{2} \rho_{\mathrm{CIGS}}\right)$. Therefore, an overlap of the layers at the interface through the thickness of the specimen perfectly agrees with the curves observed in Fig. 5c, d.

4. At the pn-junction of the solar cell (i.e., the CdS/ CIGS interface in case of a heterojunction or the topmost part of the CIGS layer in case of a buried junction) an increase of the potential by the builtin voltage is expected from the p-type towards the n-type semiconductor, i.e., from CIGS towards the $\mathrm{CdS}$ layer. When assuming a potential increase of $1 \mathrm{~V}$ a variation of roughly $7 \%$ is expected which corresponds to a phase variation of $\sim 1.2 \mathrm{rad}$ in (a) resp. $\sim$ $0.9 \mathrm{rad}$ in (b) and to an apparent thickness increase of $\sim 12 \mathrm{~nm}$ in Fig. $5 \mathrm{c}$ resp. $\sim 9 \mathrm{~nm}$ in Fig. 5 d. Indeed, especially in Fig. 5c, a blurred increase of the phase shift is observed in CIGS close below the CIGS/CdS interface in contrast to the HAADF-STEM intensity. However, the signal cannot be assigned clearly to the pn-junction. In case of a heterojunction, the signal of the pn-junction overlaps with changes of the local chemistry which strongly affect the $M I P, Z$, and $\rho_{\text {at }}$. In this case, the chemical environment may lead to much larger variations in the phase shift compared to the shift due to the pn-junction. Considering a buried junction, the EDX line profiles in Fig. 5e, $\mathrm{f}$ indicate also strong variations of the local chemistry (especially GGI) in the topmost part of CIGS. Therefore, also the signal of a buried junction could not be reliably separated from the contribution of MIP variations.

In Fig. 6a, b, a mosaic of four phase images is shown that is retrieved from the holograms acquired on the solar cell with an absorber layer with high $\mathrm{Cu}$ content (GGI $\sim 0.37$ and CGI $\sim 0.99$ ). Again, the HAADF-STEM (c) and the BF-STEM (d) images are shown and the analyzed regions are marked in each micrograph. In Fig. 7a, b, the line profiles of the phase variation and the corresponding HAADFSTEM intensity are sketched as well as the corresponding relative thickness variation ( $t_{\text {HAADF-STEM}}$ ) and the absolute thickness $\left(t_{\text {phase }}\right)($ Fig. $7 \mathrm{c}, \mathrm{d})$. Also for these phase profiles, residual background gradients were subtracted. The absolute specimen thickness, measured by the phase variation, reveals a specimen thickness in CIGS of 50-60 nm which agrees well with values measured by EFTEM, i.e., $50-80 \mathrm{~nm}$. Fig. 7e gives the EDX line profile of the region (A) and Fig. $7 f$ displays EDX line profiles acquired close to the region $\mathrm{B}$.
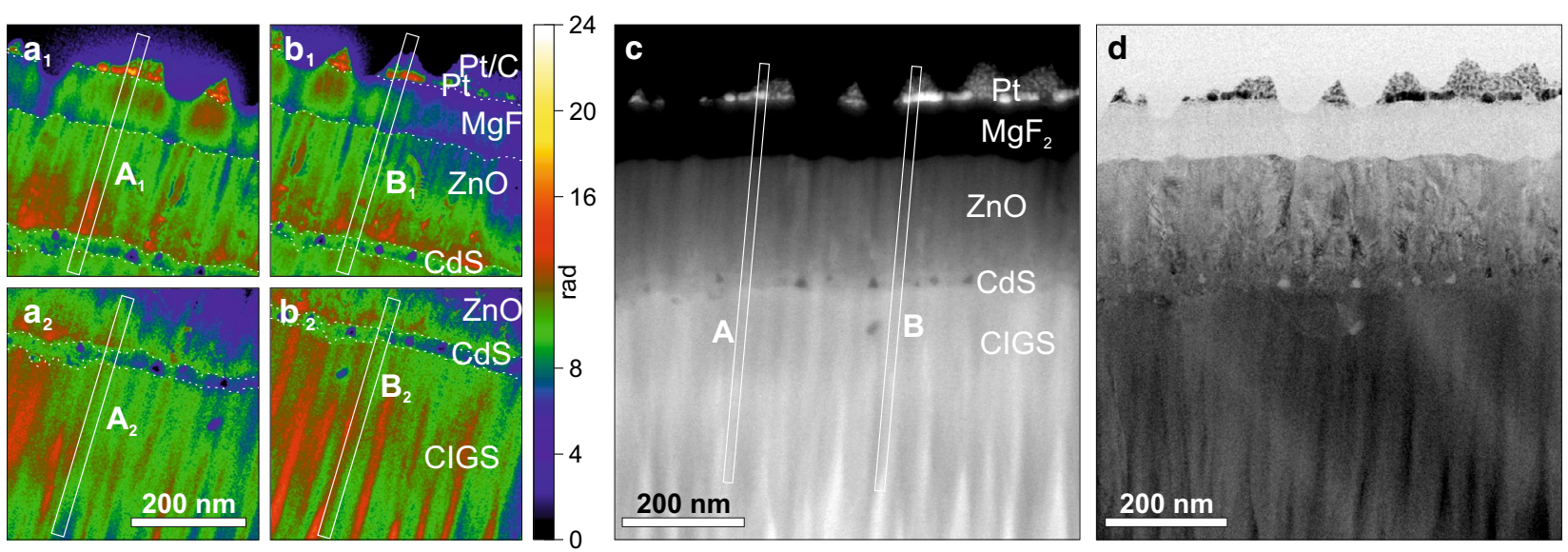

Fig. 6 Off-axis holography on solar cell with CIGS of high Cu content. a, b Extracted phase images from off-axis holograms acquired on the solar cell that has an absorber layer with high Cu content. $\mathbf{c}$ HAADF-STEM and $\mathbf{d}$ BF-STEM micrographs show the same region of the solar cell. The line profiles that were extracted from the phase images and the HAADF-STEM image along the marked areas are given in Fig. 7 

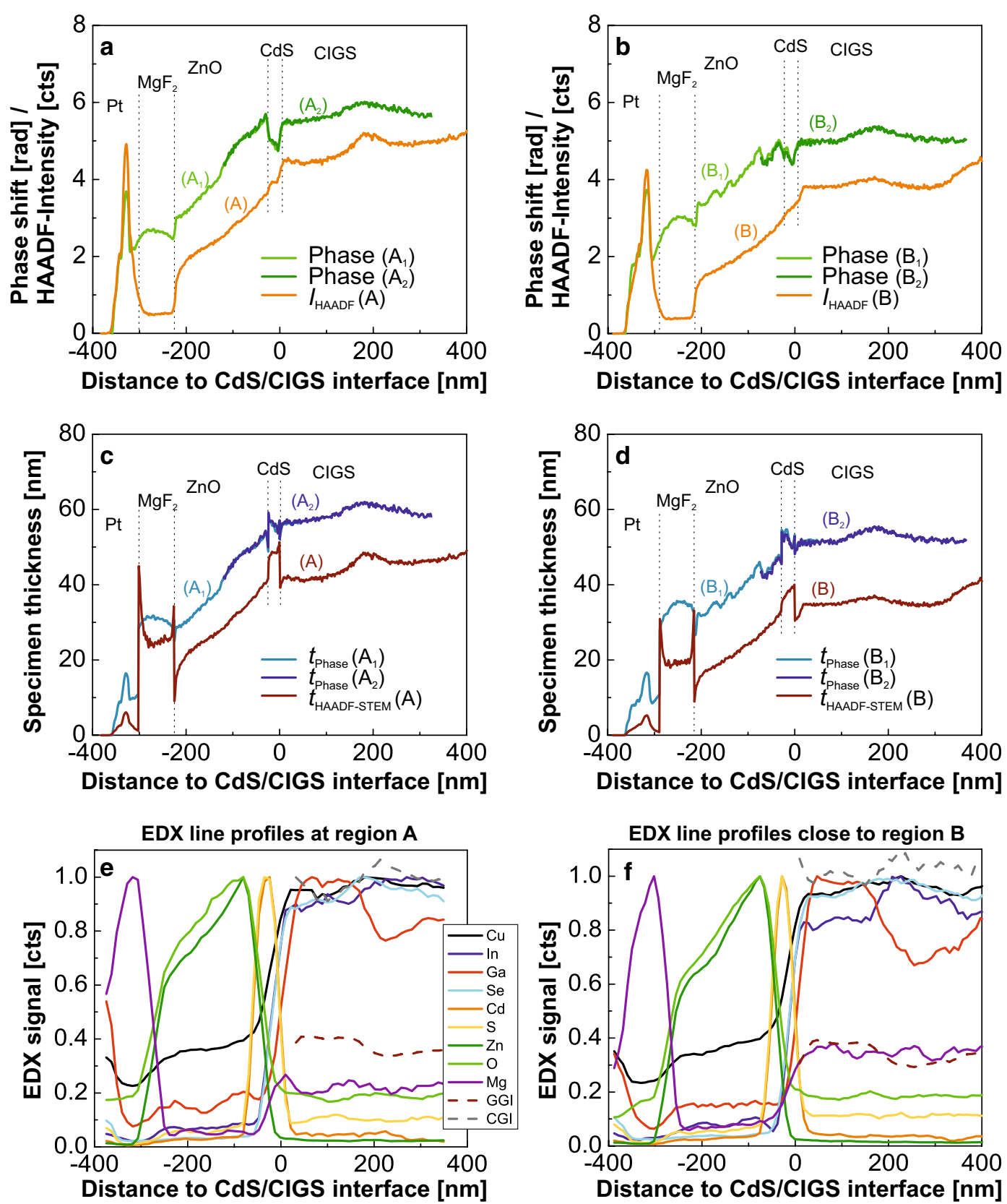

Fig. 7 Phase variations on solar cell with CIGS of high Cu content $(\mathbf{a}, \mathbf{b})$ Intensity line profiles showing the phase variation (green lines) which is extracted from the regions $A_{1}, A_{2}, B_{1}$, and $B_{2}$ marked in Fig. 6a, b. Below, the intensity profiles extracted from the same regions $(A, B)$ in the HAADFSTEM image (Fig. 6a, b), are shown (a, b, yellow lines). In order to correct for residual background gradients in the phase images, a linear background was subtracted from the phase variation line profiles $\left(A_{1}, A_{2}, B_{1}\right.$, and $\left.B_{2}\right)$. The relative thickness variation, measured by HAADF-STEM and the absolute thickness variation, measured by the phase images are given in $(\mathbf{c}, \mathbf{d})$. The EDX line profile corresponding to region $A$ is depicted in (e) showing relative concentration variations. The EDX line profile which is shown in (f) stems from a region close to $\mathrm{B}$. The copper signal in the $\mathrm{ZnO}$ region originates from stray electrons that impinge on the copper grid. Further a significant amount of $\mathrm{Mg}$ and $\mathrm{O}$ is measured in the CIGS region. These are artifacts due to the overlap of the Se L-lines with the K-lines of $\mathrm{Mg}$ and the $\mathrm{Cr}$ L-lines ( $\mathrm{Cr}$ is present in the TEM specimen holder) with the $\mathrm{O}$ K-lines. In addition to the EDX profiles of the present elements, also the relative variation of GGI and CGI is qualitatively shown in the graph

Similar as in the profiles measured on the standard solar cell, the change due to the differently doped layers in $\mathrm{ZnO}$, interface roughness in $\mathrm{CdS}$ and the pn-junction (resp. local changes in chemistry) close to the CdS/CIGS interface are found in the phase profiles on the solar cell with an absorber layer with high $\mathrm{Cu}$ content. However, in addition 
to these features, the CIGS with high Cu content shows additional variations in both, $t_{\text {phase }}$ and $t_{\text {HAADF-STEM, }}$ at a distance of $\sim 200 \mathrm{~nm}$ below the CdS/CIGS interface. As found by the corresponding EDX profiles, the GGI decays at this position. Thus it is assumed that the variation is caused by different chemical variations which are related to the high $\mathrm{Cu}$ content in CIGS compared to the CIGS with low $\mathrm{Cu}$ content, e.g., the $\mathrm{Ga}$ notch might be shifted closer towards the interface. However, as at the same time the Se signal of the EDX profiles raises at roughly 200 $\mathrm{nm}$ below the interface, a slight thickness increase of the specimen at this position cannot be excluded as well. Furthermore, compared to the standard solar cell, additional inhomogeneities appear in the $\mathrm{ZnO}$ layer of the solar cell with the absorber layer with high $\mathrm{Cu}$ content in the profile $t_{\text {phase }}$ in Fig. 7d. However, the $\mathrm{ZnO}$ layer of both solar cells is expected to be similar. Therefore, these inhomogeneities are attributed to diffraction effects which are apparent in the BF-STEM micrograph in Fig. $6 \mathrm{~d}$.

Summing up the results presented in this section, various phase variations were identified in the region containing and surrounding the pn-junction of two different solar cells, e.g., phase variations were located in the $\mathrm{ZnO}$ layer, whose positions correlate with the transition between the differently doped layers $\mathrm{i}-\mathrm{ZnO}$ and $\mathrm{ZnO} \mathrm{O}$ Al. Further, phase variations at the $\mathrm{ZnO} / \mathrm{CdS} / \mathrm{CIGS}$ interface region and in CIGS were observed. Comparing the two CIGS layers of different $\mathrm{Cu}$ content, slightly different phase variations were observed, i.e., the phase image of the $\mathrm{Cu}$-poor CIGS revealed additional, disordered short-range variations. However, the difference between the two CIGS layers is most probably related to compositional changes and not mainly to electronic inhomogeneities. $\mathrm{Cu}$-poor growth conditions not only affect the GGI variation in CIGS but may also enhance the growth of smaller, disordered grains below the CIGS/CdS interface. Thus, these differences do not necessarily reflect changes in electronic properties, but rather depend on $\triangle M I P$ and diffraction effects.

\section{Local potential variations \\ Local inhomogeneities in CdS and CIGS}

In Fig. 8, overview HAADF-STEM (a) and BF-STEM (b) images are exemplarily shown which are mosaic displays of several micrographs showing the CIGS/CdS/ $\mathrm{ZnO}$ interfaces in the solar cell with the absorber layer of high $\mathrm{Cu}$ content. Off-axis holograms were acquired in the regions A-D which are marked by red squares in Fig. 8. The corresponding retrieved phase images (Fig. 9a-d) show local inhomogeneities of smaller phase shift compared to the surrounding area. The inhomogeneities mainly appear in the CdS, especially close to the $\mathrm{CdS} / \mathrm{CIGS}$ interface and in regions where the CdS layer is thick. But also within the CIGS layer, some inhomogeneities are present, mainly along some specific grain boundaries.

Profiles extracted from the reconstructed phase images (Fig. 9a-d) show variations across such inhomogeneities in a range of $0.35-1.9 \mathrm{rad}$ in CdS (Fig. 9e, g, i, j) and 0.45-0.75 rad in CIGS (Fig. 9f, h). The drop in the phase shift may originate from (i) reduced thickness (voids) or from (ii) local decrease of the potential in this region. Therefore, the local, absolute thickness was calculated for each individual profile using the absolute phase change measured next to the inhomogeneity and the MIP values for CdS $(13.5 \mathrm{~V})$ and $\mathrm{CuIn}_{0.64} \mathrm{Ga}_{0.36} \mathrm{Se}_{2}(14.8 \mathrm{~V})$ as given in Table 1 [34]. Then, the corresponding thickness of the
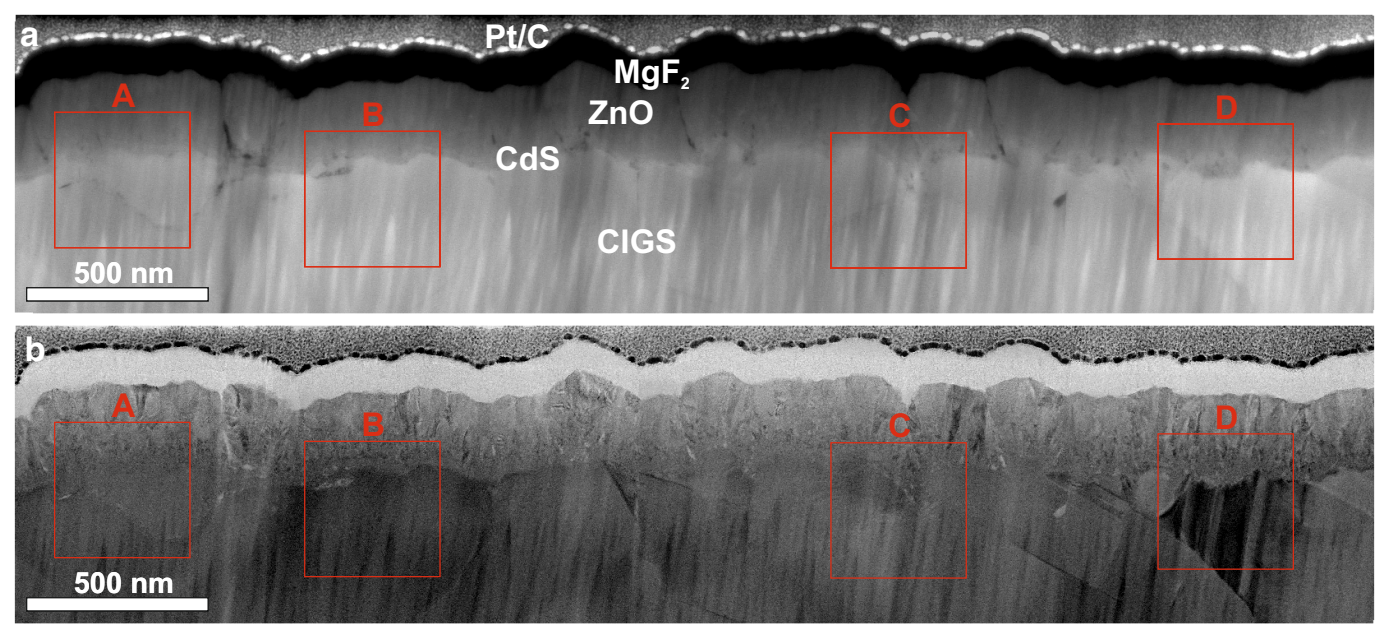

Fig. 8 Inhomogeneities in the pn-junction region. a HAADF-STEM and b BF-STEM image of the solar cell with an absorber layer with high Cu content in the $\mathrm{ZnO} / \mathrm{CdS} / \mathrm{CIGS}$ interface region. The regions marked by the red squares are analyzed by off-axis holography in Fig. 9 

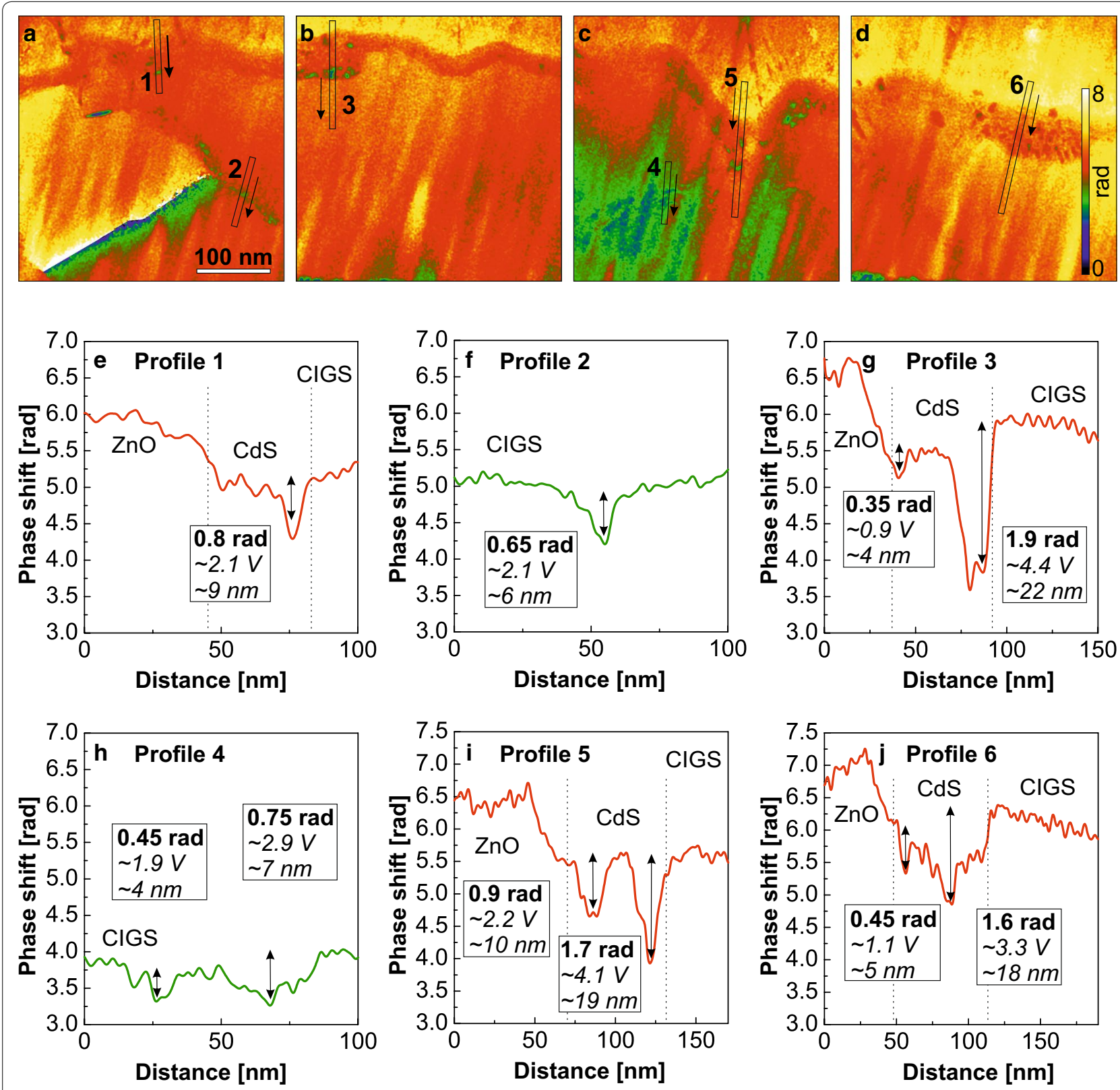

Fig. 9 Phase variations at inhomogeneities in the pn-junction region. $\mathbf{a}-\mathbf{d}$ Reconstructed phase images from selected sites labelled as $\mathbf{a}-\mathbf{d}$ in Fig. 8. Inhomogeneous regions are visible in CdS and CIGS. The line profiles (e-j) were extracted from the marked regions 1-6, whereas profiles nr. 1,3,5, and 6 show variations in CdS and profiles 2 and 4 variations in CIGS. The extent of the absolute phase variations are indicated next to the profiles. Furthermore, the corresponding potential variation needed to cause the measured phase shift under the assumption that the thickness is constant is indicated (case ii) and also the thickness variation needed to cause the phase shift under the assumption that the potential is constant (case i)

pore in case (i) and the potential variation in case (ii) were calculated based on the obtained local specimen thickness and the corresponding MIP according to Eq. (1). Both values (cases $\mathrm{i}$ and ii) are indicated in the boxes for each graph as well. Since the inhomogeneities are also visible in the HAADF-STEM image and changes in the potential of $\sim 4.4 \mathrm{~V}$ in $\mathrm{CdS}$ and $\sim 2.9 \mathrm{~V}$ in CIGS would be much larger than expected, it is supposed that the variation in the phase shift is caused by voids in the sample. In this case (i), the thickness of the measured voids would be 4-7 nm for pores along the CIGS grain boundaries and 4-22 nm for pores in CdS. The analysis of the standard solar cell with an absorber layer with low $\mathrm{Cu}$ content revealed similar inhomogeneities in CIGS and CdS (not shown). 


\section{Grain boundary in CIGS}

Potential variations were measured at a CIGS grain boundary of random orientation in the standard CIGS absorber layer (see Table 2) by in-line holography. Aiming at the evaluation of the accuracy, precision, and reliability of phase variation measurements by in-line holography and the TIE reconstruction approach, the CIGS grain boundary provides an ideal region of interest. Due to its clear recognizability, the same grain boundary was investigated by three different microscopes in order to assess factors that are related to the used instrument. The three microscopes (1) Jeol 2200FS, (2) Jeol ARM 200F, and (3) Hitachi I2TEM were used.
The phase reconstruction was based on the modified TIE described in the "Methods" section including symmetrization, Tikhonov-type filtering, 200 alignment iterations, and the NR approximation for the estimation of the derivative based on five images. The strength of the Tikhonov-type filter $\left(\mathbf{q}_{\mathrm{c}}=0.001 \AA^{-1}\right)$ was chosen by visually evaluating the strength that allows for efficiently suppressing noise but still acceptably preserving the potential well. In Fig. 10, TEM micrographs of the grain boundary in focus $(\mathrm{a}-\mathrm{c})$ as obtained with each microscope are shown as well as the finally calculated intensity derivatives $(\mathrm{d}-\mathrm{f})$ and the corresponding reconstructed phase images $(g-i)$.
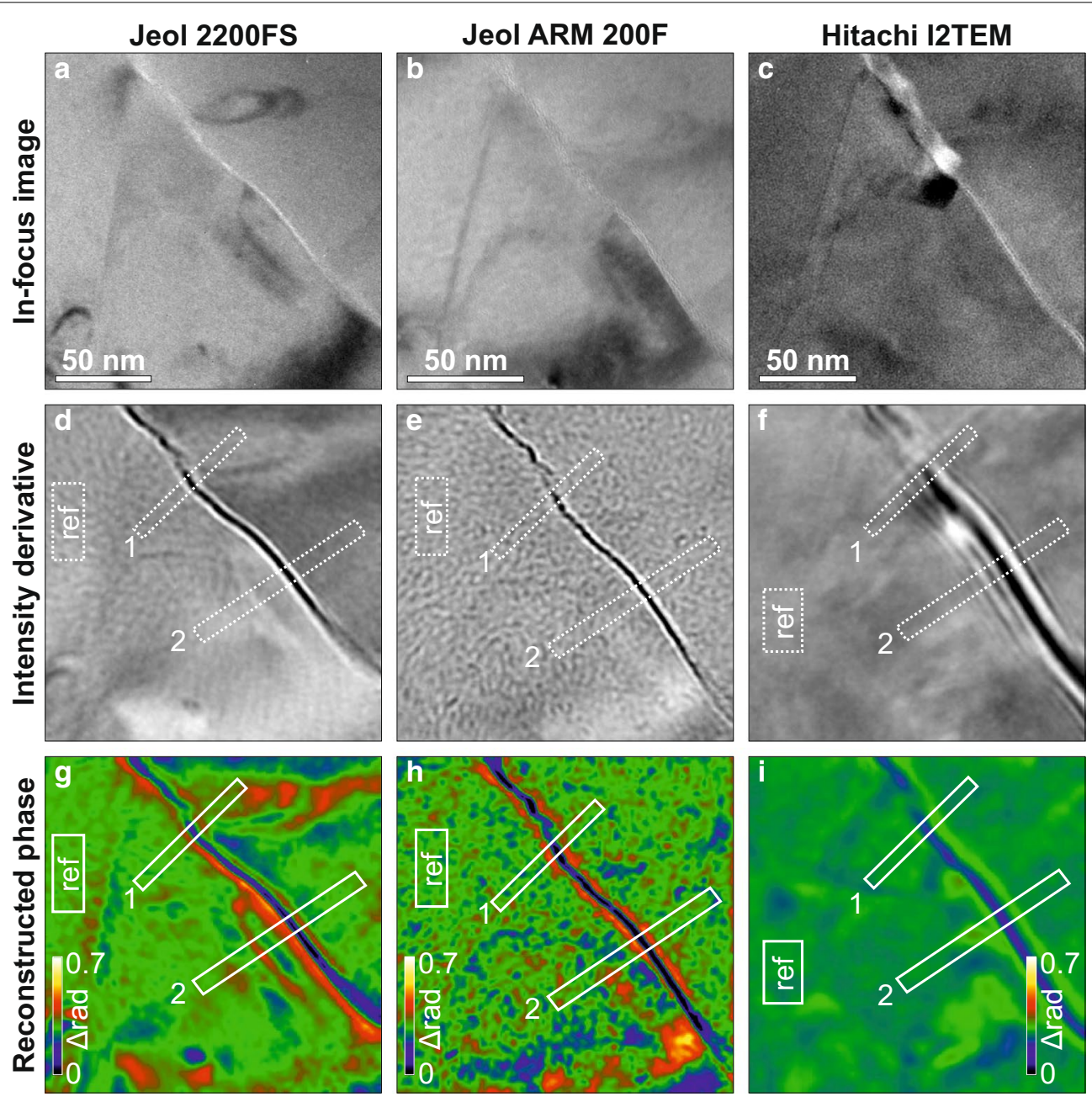

Fig. 10 In-line holography at CIGS grain boundary. a-c In-focus TEM micrographs acquired on different microscopes, $\mathbf{d}$-f intensity derivatives dl/dz obtained within the last iteration of the reconstruction procedure according to the NR approach based on five images and $\mathbf{g - i}$ the corresponding reconstructed phase images. The modified TIE as described in the methods section was applied for all reconstructions. The modifications include symmetrization, Tikhonov-type filtering with $\boldsymbol{q}_{\mathrm{C}}=0.001 \AA^{-1}, 200$ alignment iterations and the estimation of the intensity derivative according to the NR approach. The series acquired on the Jeol 2200FS and the Jeol ARM 200F contain defocus images of $\Delta z_{1}= \pm 3.2 \mu \mathrm{m}$ and $\Delta z_{2}= \pm 6.4 \mu \mathrm{m}$ and the series acquired on the Hitachi I2TEM contains images with the defoci $\Delta z_{1}= \pm 10.5 \mu \mathrm{m}$ and $\Delta z_{2}= \pm 21 \mu \mathrm{m}$. The rectangles marked as 1 and 2 in the phase images indicate the regions where the profiles shown in Fig. 11 were extracted. Furthermore, the reference area is shown where the phase shift was set to zero 
A qualitative comparison of the three focal series indicates that all reconstructions are punctually affected by diffraction related artifacts. Considering the Jeol ARM $200 \mathrm{~F}$ and the Jeol 2200FS, it was found that the use of a small objective aperture decreases diffraction effects in the focal series and improves the quality of the phase reconstruction as long as the diffraction limit does not dominate the spatial resolution. The difference between the phase images obtained by the Jeol 2200FS and the Jeol ARM 200F can thus be explained by the smaller objective aperture which was used on the Jeol ARM 200F in contrast to the Jeol 2200FS, as well as $\mathrm{C}_{\mathrm{S}}$-correction and the higher coherency of the electron source in the Jeol ARM 200F.

In order to quantitatively analyze the results, two phase profiles were extracted across the grain boundary in the regions 1 and 2 marked in Fig. 10d-f and $g-i$. The corresponding potential variations are displayed in Fig. 11. They are calculated according to Eq. (1), assuming a constant specimen thickness of $30 \mathrm{~nm}$ as it was estimated by EFTEM [46]. The depths of the measured potential wells vary within $130-1500 \mathrm{mV}$ depending on the used microscope and the position where the profile is measured. The possible sources of potential variations at grain boundaries in CIGS and their expected magnitudes are discussed in detail by Schmidt [6]. Changes in the local chemistry may cause variations in the MIP of up to a few volts. In contrast, potential variations related to electronic inhomogeneities $\left(V_{\mathrm{E}}\right)$ or lattice distortions ( $V_{\text {Dist }}$ ) are expected to be lower, i.e., $\sim 100 \mathrm{mV}$. Therefore, it is supposed that the measured potential variations mainly reflect variations of the MIP. The local MIP may be affected by (1) the degree of the ionicity of the atoms, (2) by the local composition and (3) by the atomic density, e.g., by vacancies.
1. Regarding changes in the ionicity of the atoms, the MIP considerably decreases with increasing ionicity of the cationic sites, e.g., if all $\mathrm{Cu}$-sites are ionized or $28 \%$ of the $\mathrm{Ga}$ and In sites are ionized, a potential decrease of $1 \mathrm{~V}$ is obtained. However, if charge neutrality within the unit cell is assumed, also the ionic scattering factor of Se needs to be considered. In this case, a decrease of the MIP by $1 \mathrm{~V}$ is obtained if e.g., the ionization of all atoms is increased by $69 \%$ in $\mathrm{CuIn}_{0.64} \mathrm{Ga}_{0.36} \mathrm{Se}_{2}$, considering the scattering factors based on values reported by Rez et al. [33].

2. Variations of the GGI only cause slight changes in the MIP as the change in unit cell volume compensates for the difference in the scattering factors of In and Ga. Therefore, a decrease of the MIP in the range of $1 \mathrm{~V}$ would only be reached, if the GGI increases from 0 to nearly 1 , while the lattice constant is maintained which is not expected.

3. Finally, a decrease of the $M I P$ by $1 \mathrm{~V}$ can be caused by decreasing the average atomic density by $8 \%$. Regarding $\mathrm{Cu}$ vacancies, a decrease of the MIP by $1 \mathrm{~V}$ could be achieved only by turning roughly a third of all the $\mathrm{Cu}$ sites into vacancies, while maintaining the lattice constant. However, taking into account that the crystal lattice is interrupted at the grain boundary, an additional decrease of the average atomic density at the grain boundary is expected which may fully or partly be responsible for the measured fluctuations.

Compared to values reported in literature which are in the range of $1-3 \mathrm{~V}$ for random grain boundaries [6, 27], most of the experimentally determined values in this work are lower than expected. Especially, the potential wells measured using the Hitachi I2TEM are considerably weaker than expected and differ from the values
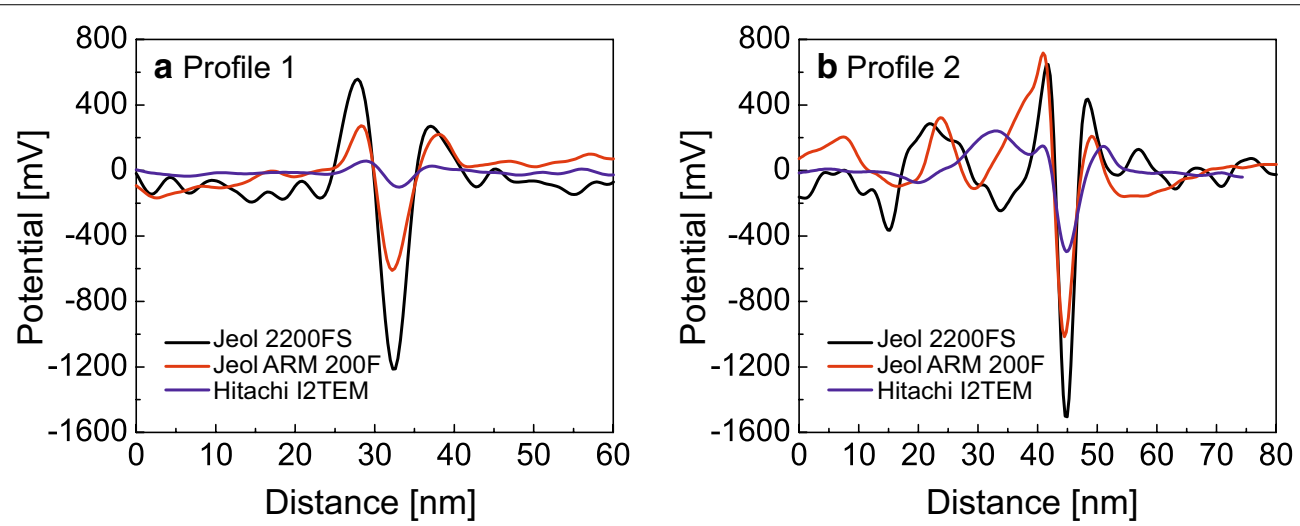

Fig. 11 Potential wells at CIGS grain boundary. Phase variation profiles extracted from the phase images in Fig. 10d-f, i.e., from the indicated region 1 (a) and from the indicated region 2 (b) of the grain boundary. Potential wells in the range of 130-1500 mV are measured depending on the used microscope and the position of the grain boundary from where the profile is extracted 
measured using the Jeol 2200FS and the Jeol ARM 200F. However, various factors limit the accuracy of the variations which may explain these discrepancies.

Firstly, the absolute values are affected by factors whose values are not precisely known but that linearly affect the calculation of the potential well, i.e., the specimen thickness and the magnitude of the real defocus step. Inaccuracies in the estimated specimen thickness lead to a systematic error which is independent from the microscope and from the phase retrieval algorithm. In the present work, an error of the thickness estimation in the range of $\pm 20 \%$ is expected. Further, according to Eq. (8), inaccuracies in the estimation of the real defocus step impact the result. Also, as the measurement reveals the total potential variation which is averaged across the specimen thickness, the determined variation is only accurate if the potential variation is uniform within the specimen thickness. Analyzing a slightly mistilted or an uneven grain boundary plane thus leads to an apparently broader and shallower potential well [6].

Secondly, artifacts related to the TIE such as the Tikhonov-type filter, the intensity derivative approximation or artifacts such as diffraction effects impact the measured, absolute potential variations: The use of a too strong Tikhonov-type filter causes damping of the phase variation. In the present work, it is assumed that filterrelated damping is in the range of 10-30\%. Further, also the choice of the defocus steps affects the accuracy of the phase reconstruction, as the intensity derivative is an approximation limited by noise (small defocus steps) and artifacts due to non-linearity (large defocus steps). In practice, the choice of defocus further determines the amount of image misalignment and distortions. The larger the defocus differences, the larger are the differences of the images in the focal series. This unavoidably enhances the error due to image misalignments (including image rotation and changes in magnification) [24]. Thus, to estimate the effect of the chosen defocus step, a (perfectly aligned) focal series was simulated according to [47] based on the amplitude image in Fig. 10a and the phase image in Fig. 10g. Retrieving the phase again from this simulated series revealed that the absolute potential variations are only slightly affected by the choice of the defocus step in the present defocus range $(1-10 \mu \mathrm{m})$. However, if the simulated image stack is misaligned by applying a random shift to each image, the reconstructed potential variation gets strongly damped with increasing defocus steps. Considering the experimentally determined potential wells, where residual misalignment of the image stack is present, the same trend is observed as well (Fig. 12): The measured depth of the potential well at the grain boundary decreases with increasing defocus steps. This trend was observed

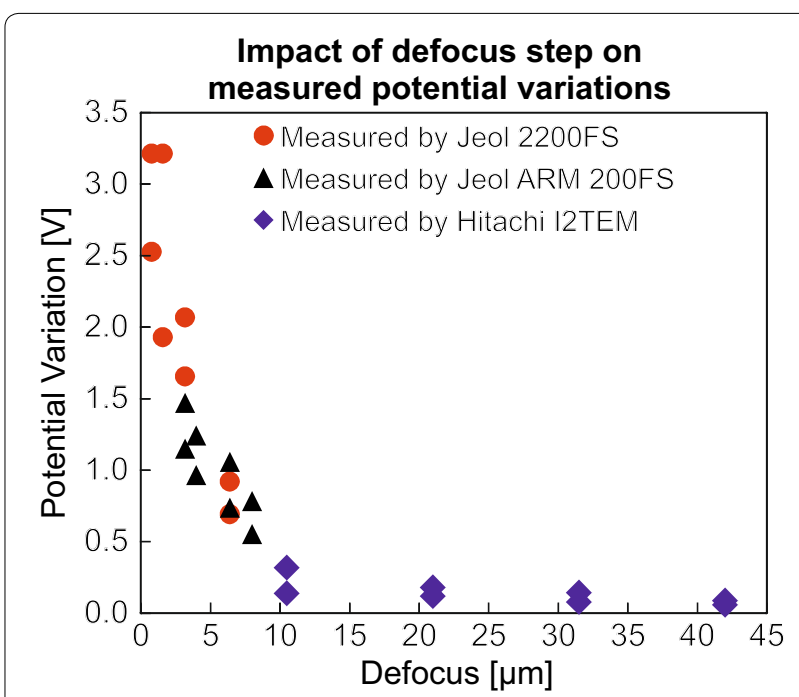

Fig. 12 Effect of defocus step. Experimentally measured potential well depths depend on the applied defocus step (the reconstruction is performed using three images only). Although, the difference of the potential variation due to defocus variation is not the same for different microscopes, a clear trend is visible in the graph. The measured potential well depth decreases with increasing defocus step in a non-linear dependency. As the remaining misalignment of the focal series becomes more severe with increasing defocus steps, the resulting potential variation is damped more strongly leading to an underestimation of the real potential well if large defocus steps are applied. In contrast, if small defocus steps are used, the accuracy is limited by the effects of noise. Therefore, a direct comparison of results obtained on different microscopes or based on different defocus steps is not possible

on all three microscopes. Hence, the potential well measured by the Hitachi I2TEM appears to be smaller compared to the results obtained by the Jeol 2200FS and Jeol ARM 200F, because the used defocus steps in the Hitachi I2TEM are significantly larger $(\sim 21 / 10.5 \mu \mathrm{m})$ than in the Jeol 2200FS and the Jeol ARM 200F (both $\sim 6.4 / 3.2 \mu \mathrm{m}$ ). Besides this, also the different acquisition mode (holograms) used with the Hitachi I2TEM, differences in the diffraction effects or stronger deviations from the edge-on orientation of the grain boundary may further decrease the resulting potential well and thus contribute to the deviation between the results obtained by the different microscopes. Especially, the results obtained by the Hitachi I2TEM at profile 1 may be affected by diffraction effects as strong artifacts are caused by the small grain close to the profile (see e.g., Fig. 10c) which may damp the measured potential well resulting in the comparably low value of $130 \mathrm{mV}$. Taking all the different limitations into account, a comparison of results obtained on different microscopes or obtained with different defocus steps is in general not possible straightforwardly. 


\section{Analysis of resolution with respect to electronic characterization}

The analysis of long- and short-range potential variations in CIGS solar cells revealed the presence of various inhomogeneities in the investigated layers $\mathrm{ZnO}, \mathrm{CdS}$, and CIGS. However, in general the measured potential fluctuations are predominantly caused by MIP inhomogeneities, whereas no fluctuations could clearly be attributed to variations in the electronic properties $\left(V_{\mathrm{E}}\right)$. Therefore, the gained information is mainly of chemical nature and does not reveal direct information on fluctuations in the band structure. Considering potential variations caused by redistribution of free charge carriers, contributions of a few hundred millivolts are expected for high-efficiency CIGS solar cells, except at the pn-junction where variations in a range of $1 \mathrm{~V}$ are reasonable. Therefore, a high phase resolution is crucial for analyzing electronic properties by holography.

The theoretical phase detection limit $\delta \phi$ of an off-axis hologram is determined according to Eq. (10) by the fringe contrast $\mu$ and the number of counts per pixel $N$ or the acquisition time $t$, respectively [48].

$$
\delta \phi=\frac{\sqrt{2}}{\mu \sqrt{N}} \propto \frac{\sqrt{2}}{\mu \sqrt{t}} .
$$

The fringe contrast is determined by the electron beam spread and the degree of coherence of the electron source. Therefore, the theoretical phase detection limit depends mainly on the microscope and sets a fixed limitation to the hologram quality and phase resolution for a certain spatial resolution. In addition, the phase reconstruction procedure includes a low-pass window function which further deteriorates the reachable phase resolution for a certain spatial resolution [49]. In literature, a decrease of the signal-to-noise ratio (SNR) by roughly one order of magnitude was reported for medium (spatial) resolution holography [50]. The present off-axis holograms show around 2500 counts per pixel (respectively 125 electrons per pixel) and a contrast of $13.8 \%$. Therefore, the theoretical phase detection limit is $\sim 2.0 \mathrm{mrad}$ according to Eq. (10). In dependence of the required spatial resolution, the phase resolution further deteriorates, as is shown in Fig. 13a. Thus, aiming for a spatial resolution of $5-10 \mathrm{~nm}$, the reconstructed phase images achieve a phase resolution of roughly $0.2 \mathrm{rad}$. Based on the present parameters, the conditions (specimen thickness, acquisition time, fringe contrast) under which an inhomogeneity could be identified, are extrapolated. In Fig. 13, these conditions are shown for the two cases where a potential resolution of (b) $1 \mathrm{~V}$ or (c) 100 $\mathrm{mV}$ is required, with both at an SNR of 3. A fluctuation of $1 \mathrm{~V}$, i.e., in the range of the pn-junction in CIGS, could therefore only be detected under the current conditions with an SNR of 3, if the vertical expansion of the potential change in the TEM is around $90 \mathrm{~nm}$. However, if the expansion of the potential change is $20 \mathrm{~nm}$, the acquisition time should be increased to around $100 \mathrm{~s}$ to resolve the fluctuation. Furthermore, if weaker potential fluctuations of around $100 \mathrm{mV}$ are investigated, the conditions become more stringent to resolve the variations. Under the current conditions, such a fluctuation can only be

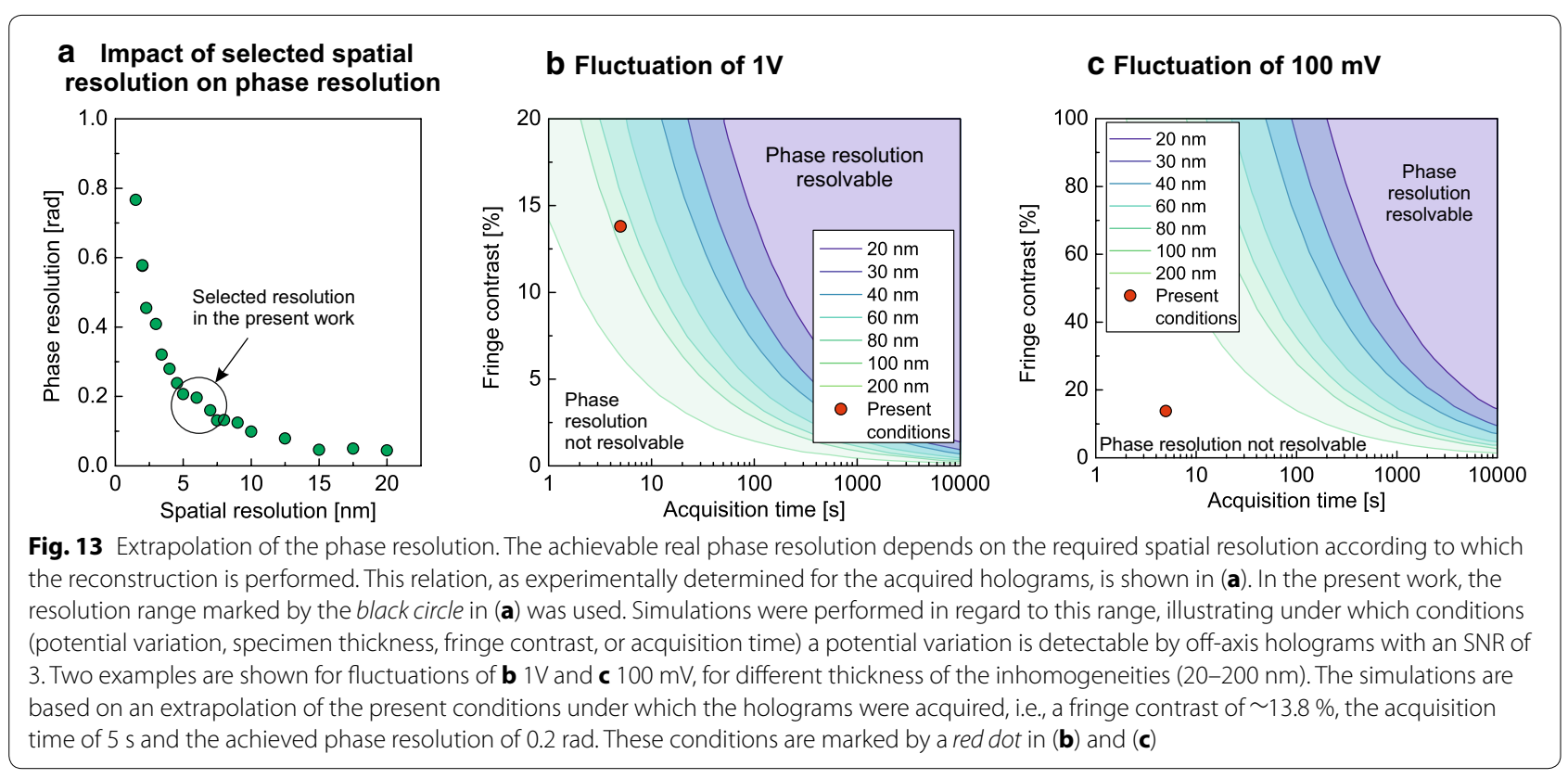


resolved if its vertical extension (and therefore also the TEM specimen thickness) is much higher than $20 \mathrm{~nm}$. In case the expansion of the fluctuation is only $40 \mathrm{~nm}$ and if the contrast could be improved to $20 \%$, still an acquisition time of $\sim 21$ min would be required to resolve the phase shift. For such a long acquisition time, the camera is likely saturated by its dark current. Thus, by optimizing the parameters for a specific problem (field of view, contrast, acquisition time, and specimen thickness), the phase resolution still needs to be improved. Nevertheless, resolving potential variations in the range of $100 \mathrm{mV}$ with nanoscale expansion $(<100 \mathrm{~nm})$ remains challenging.

The phase resolution which can be achieved by in-line holography is theoretically determined by (1) the choice of defocus which limits the frequency range that may be transferred, and (2) the degree of noise which is present in the initial images. However, in practice, also the experimental limitations as well as the reconstruction procedure play a major role. The phase resolution of the presented phase images was estimated by analyzing a region where the phase shift is assumed to be constant (i.e., within a homogeneous region of a CIGS grain). The phase detection limit was then determined as the standard deviation of the noise within this reference region. Based on this analysis, the phase images obtained by inline holography revealed a resolution of $0.05 \mathrm{rad}$ on the Jeol ARM 200F (200 kV) which corresponds to $\sim 660 \mathrm{mV}$ when considering a sample thickness of $30 \mathrm{~nm}$ and a SNR of 3 . On the Jeol 2200FS (200 kV) a phase resolution of roughly $0.03 \mathrm{rad}$ is reached which corresponds to $\sim 400$ $\mathrm{mV}(\mathrm{SNR}=3$, thickness $=30 \mathrm{~nm})$. Considering the SNR in the phase images obtained by the Hitachi I2TEM (300 $\mathrm{kV}$ ), a resolution of $\sim 0.0035 \mathrm{rad}$ is observed which corresponds to $50 \mathrm{mV}$. As the series acquired by the Hitachi I2TEM contains much larger defocus steps, compared to the Jeol ARM 200F and the Jeol 2200FS, a much better phase resolution is achieved. However, the enhanced precision or sensitivity comes at the expense of accuracy. It is therefore supposed that the phase resolution on the Hitachi I2TEM would approach a comparable range, if a focal series of smaller defocus steps would be used. Nevertheless, the achieved resolution nearly reaches the limit for investigating fluctuations in the range of $100 \mathrm{mV}$. By further increasing the exposure time, the resolution might be pushed further to the limit. In addition, more advanced iterative reconstruction procedures may be applied (see e.g., [24]) to make use of large, non-linear focal series that allows an optimized transfer of different frequency ranges and an improved image alignment algorithm.

Nevertheless, when addressing specific fluctuations of electronic properties in CIGS, one has to keep in mind that reaching the needed phase resolution is not the only issue. As discussed for long- and short-range potential variations, one main question is to separate different contributions that may lead to potential variations (see Eq. 2). In CIGS, fluctuations in electronic properties are supposed to be accompanied with compositional, chemical (ionicity), or structural variations (e.g., density of atoms and specimen thickness) which cause in general much stronger changes of the MIP than the expected change in $V_{\mathrm{E}}$. Further, thickness effects may lead to misinterpretation of measured phase variations. In principle, the measured phase variation can be corrected for thickness effects that cause a proportional increase of the phase shift with increasing specimen thickness (see Eq. (1)). However, as recently reported, also the MIP may vary significantly and non-linearly with changing specimen thickness due to different surface effects [51]. Thus, taking these issues into account makes the isolation of the phase shift contribution of $V_{\mathrm{E}}$ and therefore electronic characterization of CIGS by EH challenging.

\section{Conclusions}

Off-axis and in-line electron holography have been applied to map potential variations in CIGS thin-film solar cells on different length scales. Potential variations across the upper layers of the solar cell (i.e., $\mathrm{ZnO}$, $\mathrm{CdS}$ and CIGS) were identified by off-axis holography. This revealed various potential variations such as fluctuations in the $\mathrm{ZnO}$ layer correlating with a change of the doping type in the $\mathrm{ZnO}$ layer, and variations at the CIGS/CdS interface which indicate mainly chemical or compositional inhomogeneities. In the absorber layer, no fluctuations that could clearly be attributed to electronic inhomogeneities were observed. Close to the CdS/CIGS interface, we identified pores whose formation mechanism and impact on the device properties is not yet understood.

In-line holography was performed on a random grain boundary in CIGS using a modified TIE approach. The analysis exhibited a potential well in the range of 130$1500 \mathrm{mV}$ at the grain boundary, revealing the presence of chemical or compositional inhomogeneities. Further, the influence of the used microscope on the in-line holography results is investigated by comparing the results obtained with three different TEMs. It is found that not only the used instrument but also the chosen defocus step combined with focal series misalignment impacts the results significantly.

Finally, the resolution limits of both methods are discussed, especially in view of potential measurements by holography for electronic characterization of CIGS in the future. No potential fluctuations that are clearly related to electronic inhomogeneities could be identified in bulk CIGS. Thus, possible fluctuations are smaller than the achieved resolution (in the range of $1 \mathrm{~V}$, depending on 
the area). Thus, the results confirm the expectations, that potential variations in the investigated high efficiency CIGS solar cells are smaller than $\sim 1 \mathrm{~V}$. In order to analyze probable electronic inhomogeneities in the range of $100 \mathrm{mV}$ in CIGS solar cells by holography, not only the phase resolution needs to be further improved, but also the separation of different sources that cause phase shifts and their interpretation need to be addressed in more detail.

\section{Authors' contributions}

$D K, R E, S B, P R, F P$, and ANT designed the research and experiments. PR fabricated the solar cells, DK the TEM specimens. DK and RE performed the experiments on the Jeol FS 2200, MDR performed the experiments on the Jeol ARM 2200F. ES and CG performed the experiments on the Hitachi I2TEM. DK and RE wrote the TIE script and evaluated the in-line and off-axis holography results whereas $\mathrm{SB}, \mathrm{PR}, \mathrm{FP}$, and $\mathrm{ANT}$ assisted the interpretation considering the properties of solar cells and ES and CG assisted the evaluation of off-axis holography results. DK wrote the manuscript supervised by RE, SB, and ANT. All authors discussed, revised, and approved the manuscript. All authors read and approved the final manuscript.

\section{Author details}

${ }^{1}$ Laboratory for Thin Films and Photovoltaics, Empa - Swiss Federal Laboratories for Materials Science and Technology, Ueberlandstrasse 129, 8600 Duebendorf, Switzerland. ${ }^{2}$ Electron Microscopy Center, Empa - Swiss Federal Laboratories for Materials Science and Technology, Ueberlandstrasse 129, 8600 Duebendorf, Switzerland. ${ }^{3}$ CNRS CEMES, 29, rue Jeanne Marvig, 31055 Toulouse, France.

\section{Acknowledgements}

This work was supported by the Swiss National Science Foundation (SNF) (Project No. 200020_132377), by the European Union under the Seventh Framework Program under a contract for an Integrated Infrastructure Initiative (Reference 312483-ESTEEM2) and by the Scientific Center for Optical and Electron Microscopy (ScopeM) of the Swiss Federal Institute of Technology ETHZ. PR acknowledges the EU-FP7 R2R-CIGS project 283974. ES and CG acknowledge the "Conseil Regional Midi-Pyrnes" and the European FEDER for financial support within the CPER program, and the French National Research Agency under the "Investissement d'Avenir" program reference No. ANR-10-EQPX-38-01.

\section{Competing interests}

The authors declare that they have no competing interests.

Received: 17 November 2015 Accepted: 23 December 2015 Published online: 13 January 2016

\section{References}

1. Jackson, P., Hariskos, D., Wuerz, R., Kiowski, O., Bauer, A., Friedlmeier, T.M., Powalla, M.: Properties of $\mathrm{Cu}(\mathrm{In}, \mathrm{Ga}) \mathrm{Se} 2$ solar cells with new record efficiencies up to $21.7 \%$. Physica status solidi (RRL) - Rapid Res Lett $\mathbf{9}(1)$, 28-31 (2015). doi:10.1002/pssr.201409520

2. Werner, J.H., Mattheis, J., Rau, U.: Efficiency limitations of polycrystalline thin film solar cells: case of $\mathrm{Cu}(\mathrm{In}, \mathrm{Ga}) \mathrm{Se}$. Thin Solid Films 480-481, 399-409 (2005). doi:10.1016/j.tsf.2004.11.052

3. Siebentritt, S.: What limits the efficiency of chalcopyrite solar cells? Solar Energy Mat Solar Cells 95(6), 1471-1476 (2011). doi:10.1016/j. solmat.2010.12.014

4. Wei, S.-H., Zhang, S.B., Zunger, A.: Effects of Ga addition to CulnSe2 on its electronic, structural, and defect properties. Appl Phys Lett 72 (24), 3199-3201 (1998). doi:10.1063/1.121548

5. Han, S.-H., Hasoon, F.S., Pankow, J.W., Hermann, A.M., Levi, D.H.: Effect of Cu deficiency on the optical bowing of chalcopyrite Culn1-xGaxSe2. Appl Phys Let 87(15), 151904-1519043 (2005). doi:10.1063/1.2089154
6. Schmidt, S.S.: Microscopic properties of grain boundaries in $\mathrm{Cu}(\mathrm{In}, \mathrm{Ga}) \mathrm{Se} 2$ and CulnS2 thinfilm solar cells studied by transmission electron microscopy. PhD thesis, HZB-Berichte (2011). doi: 10.5442/d0012

7. Siebentritt, S., Igalson, M., Persson, C., Lany, S.: The electronic structure of chalcopyrites-bands, point defects and grain boundaries. Prog Photovol Res Appl 18(6), 390-410 (2010). doi:10.1002/pip.936

8. Abou-Ras, D., Schaffer, B., Schaffer, M., Schmidt, S.S., Caballero, R., Unold, T.: Direct insight into grain boundary reconstruction in Polycrystalline $\mathrm{Cu}(\mathrm{In}$, Ga)Se2 with Atomic Resolution. Phys Rev Lett 108(7), 075502 (2012). doi:10.1103/PhysRevLett.108.075502

9. Choi, P.-P., Cojocaru-Mirédin, O., Wuerz, R.: Compositional gradients and impurity distributions in CulnSe2 thin-film solar cells studied by atom probe tomography. Surf Interf Anal 44(11-12), 1386-1388 (2012). doi:10.1002/sia.4948

10. Gabor, D.: A new microscopic principle. Nature 161(4098), 777-778 (1948). doi:10.1038/161777a0

11. Rau, W.D., Schwander, P., Baumann, F.H., Höppner, W., Ourmazd, A.: Twodimensional mapping of the electrostatic potential in transistors by electron holography. Phys Rev Lett 82(12), 2614-2617 (1999). doi:10.1103/ PhysRevLett.82.2614

12. Twitchett-Harrison, A.C., Dunin-Borkowski, R.E., Midgley, P.A.: Mapping the electrical properties of semiconductor junctions-the electron holographic approach. Scanning 30(4), 299-309 (2008). doi:10.1002/ sca.20125

13. Wang, Y.G., Dravid, V.P.: Determination of electrostatic characteristics at a 24 [001] tilt grain boundary in a $\mathrm{SrTiO} 3$ bicrystal by electron holography. Philos Mag Lett 82(8), 425-432 (2002). doi:10.1080/09500830210146461

14. Chakraborty, P.S., McCartney, M.R., Li, J., Gopalan, C., Singisetti, U., Goodnick, S.M., Thornton, T.J., Kozicki, M.N.: Electron holographic characterization of nanoscale charge distributions for ultra shallow PN junctions in Si. Physica E: Low-dimensional Systems and Nanostructures 19(1-2), 167-172 (2003). doi:10.1016/S1386-9477(03)00302-3

15. Gan, Z., Perea, D.E., Yoo, J., Picraux, S.T., Smith, D.J., McCartney, M.R.: Mapping electrostatic profiles across axial p-n junctions in Si nanowires using off-axis electron holography. Appl Phys Lett 103(15), 153108 (2013). doi:10.1063/1.4824775

16. Yazdi, S., Kasama, T., Beleggia, M., Samaie Yekta, M., McComb, D.W., Twitchett-Harrison, A.C.: Towards quantitative electrostatic potential mapping of working semiconductor devices using off-axis electron holography. Ultramicroscopy 152C, 10-20 (2014). doi:10.1016/j.ultramic.2014.12.012

17. McCartney, M.R., Ponce, F.A., Cai, J., Bour, D.P.: Mapping electrostatic potential across an AIGaN/InGaN/AIGaN diode by electron holography. Appl Phys Lett 76(21), 3055-3057 (2000). doi:10.1063/1.126577

18. Cai, J., Ponce, F.A.: Determination by Electron Holography of the Electronic Charge Distribution at Threading Dislocations in Epitaxial GaN. Physica status solidi (a) 192(2), 407-411 (2002). doi:10.1002/1521-396X(200208)

19. Chung, S., Johnson, S.R., Ding, D., Zhang, Y.-H., Smith, D.J., McCartney, M.R.: Quantitative dopant profiling of $p-n$ junction in InGaAs/AlGaAs lightemitting diode using off-axis electron holography. J Vacuum Sci Technol B 28(1), 1-11114 (2010). doi:10.1116/1.3244575

20. Petersen, T.C., Keast, V.J., Paganin, D.M.: Quantitative TEM-based phase retrieval of $\mathrm{MgO}$ nano-cubes using the transport of intensity equation. Ultramicroscopy 108(9), 805-815 (2008). doi:10.1016/j. ultramic.2008.01.001

21. Donnadieu, P., Lazar, S., Botton, G.A., Pignot-Paintrand, I., Reynolds, M., Perez, S.: Seeing structures and measuring properties with transmission electron microscopy images: A simple combination to study size effects in nanoparticle systems. Appl Phys Lett 94(26), 263116 (2009). doi:10.1063/1.3168525

22. Volkov, V.V., Zhu, Y., De Graef, M.: A new symmetrized solution for phase retrieval using the transport of intensity equation. Micron 33(5), 411-416 (2002). doi:10.1016/S0968-4328(02)00017-3

23. Beleggia, M., Schofield, M.A., Volkov, V.V., Zhu, Y.: On the transport of intensity technique for phase retrieval. Ultramicroscopy 102(1), 37-49 (2004). doi:10.1016/j.ultramic.2004.08.004

24. Koch, C.T.: Towards full-resolution inline electron holography. Micron 63 69-75 (2014). doi:10.1016/j.micron.2013.10.009

25. Bhattacharyya, S., Koch, C.T., Rühle, M.: Projected potential profiles across interfaces obtained by reconstructing the exit face wave function from through focal series. Ultramicroscopy 106(6), 525-538 (2006). doi:10.1016/j.ultramic.2006.01.007 
26. Zhang, X., Oshima, Y.: Practical procedure for retrieval of quantitative phase map for two-phase interface using the transport of intensity equation. Ultramicroscopy 158, 49-55 (2015). doi:10.1016/j. ultramic.2015.06.015

27. Abou-Ras, D., Schmidt, S.S., Caballero, R., Unold, T., Schock, H.-W., Koch, C.T., Schaffer, B., Schaffer, M., Choi, P.-P., Cojocaru-Mirédin, O.: Confined and chemically flexible grain boundaries in polycrystalline compound semiconductors. Adv Energ Mat 2(8), 992-998 (2012). doi:10.1002/ aenm.201100764

28. Dietrich, J., Abou-Ras, D., Schmidt, S.S., Rissom, T., Unold, T., CojocaruMirédin, O., Niermann, T., Lehmann, M., Koch, C.T., Boit, C.: Origins of electrostatic potential wells at dislocations in polycrystalline $\mathrm{Cu}(\mathrm{In}, \mathrm{Ga})$ Se2 thin films. J Appl Phys 115(10), 103507 (2014). doi:10.1063/1.4867398

29. Lehmann, M., Lichte, H.: Tutorial on off-axis electron holography. Microsc Microanal 8(06), 447-466 (2002). doi:10.1017/S1431927602020147

30. Paganin, D., Nugent, K.A.: Noninterferometric phase imaging with partially coherent light. Phys Rev Lett 80(12), 2586-2589 (1998). doi:10.1103/ PhysRevLett.80.2586

31. Martin, A.V., Chen, F.-R., Hsieh, W.-K., Kai, J.-J., Findlay, S.D., Allen, L.J.: Spatial incoherence in phase retrieval based on focus variation. Ultramicroscopy 106(10), 914-924 (2006). doi:10.1016/j.ultramic.2006.04.008

32. Cooper, D.: Active dopant profiling in the TEM by off-axis electron holography. In: Claverie, A., Mouis, M. (eds.) Transmission electron microscopy in micro-nanoelectronics, p. 264. John Wiley and Sons, Inc., Hoboken. (2012)

33. Rez, D., Rez, P., Grant, I.: Dirac-Fock calculations of X-ray scattering factors and contributions to the mean inner potential for electron scattering. Acta Crystallographica Sect A Found Crystallograph 50(4), 481-497 (1994). doi:10.1107/S0108767393013200

34. Weickenmeier, A., Kohl, H.: Computation of absorptive form factors for high-energy electron diffraction. Acta Crystallographica Sect A Foundation Crystallograph 47(5), 590-597 (1991). doi:10.1107/ S0108767391004804

35. Peng, L.-M.: Electron scattering factors of ions and their parameterization. Acta Crystallographica Sect A Found Crystallograp 54(4), 481-485 (1998). doi:10.1107/S0108767398001901

36. Chirilă, A., Buecheler, S., Pianezzi, F., Bloesch, P., Gretener, C., Uhl, A.R., Fella, C., Kranz, L., Perrenoud, J., Seyrling, S., Verma, R., Nishiwaki, S., Romanyuk, Y.E., Bilger, G., Tiwari, A.N.: Highly efficient Cu(In, Ga)Se2 solar cells grown on flexible polymer films. Nat Mat 10(11), 857-861 (2011). doi:10.1038/ nmat3122

37. Chirilă, A., Reinhard, P., Pianezzi, F., Bloesch, P., Uhl, A.R., Fella, C., Kranz, L., Keller, D., Gretener, C., Hagendorfer, H., Jaeger, D., Erni, R., Nishiwaki, S., Buecheler, S., Tiwari, A.N.: Potassium-induced surface modification of $\mathrm{Cu}(\mathrm{In}, \mathrm{Ga}) \mathrm{Se} 2$ thin films for high-efficiency solar cells. Nat Mat 12(12), 1107-1111 (2013). doi:10.1038/nmat3789

38. Teague, M.R.: Deterministic phase retrieval: a Green's function solution. J Optical Soc Am 73(11), 1434-1441 (1983). doi:10.1364/JOSA.73.001434
39. De Graef, M.: Lorentz microscopy: Theoretical basis and image simulations. In: Zhu, MDGaY (ed.) Experimental Methods in the Physical Sciences. Magnetic Imaging and Its Applications to Materials, vol. 36, pp. 27-67. Academic Press, London (2001). doi:10.1016/ S1079-4042(01)80036-9

40. McVitie, S., Ngo, D.T:: Quantitative measurements of phase using the transport of intensity equation. J Phys Conf Series 126(1), 012041 (2008). doi:10.1088/1742-6596/126/1/012041

41. Ishizuka, K., Allman, B.: Phase measurement of atomic resolution image using transport of intensity equation. J Electron Microsc 54(3), 191-197 (2005). doi:10.1093/jmicro/dfi024

42. Barty, A., Paganin, D., Nugent, K.: Phase retrieval in lorentz microscopy. In: Zhu, Y., De Graef, M. (eds.) Experimental Methods in the Physical Sciences. Magnetic Imaging and Its Applications to Materials, vol. 36, pp. 137-166. Academic Press, London (2001). 10.1016/S1079-4042(01)80039-4

43. Zhang, X., Oshima, Y.: Experimental evaluation of spatial resolution in phase maps retrieved by transport of intensity equation. Microscopy, 045 (2015). doi: 10.1093/jmicro/dfv045

44. Waller, L., Tian, L., Barbastathis, G.: Transport of Intensity phase-amplitude imaging with higher order intensity derivatives. Optics Express 18(12), 12552-12561 (2010). doi:10.1364/OE.18.012552

45. Soto, M., Acosta, E.: Improved phase imaging from intensity measurements in multiple planes. Appl Optics 46(33), 7978-7981 (2007). doi:10.1364/AO.46.007978

46. Malis, T., Cheng, S.C., Egerton, R.F.: EELS log-ratio technique for specimenthickness measurement in the TEM. J Electron Microsc Tech $\mathbf{8}(2), 193-200$ (1988). doi:10.1002/jemt.1060080206

47. Erni, R., Rossell, M.D., Nakashima, P.N.H.: Optimization of exit-plane waves restored from HRTEM through-focal series. Ultramicroscopy 110(2), 151-161 (2010). doi:10.1016/j.ultramic.2009.10.015

48. Harscher, A., Lichte, H.: Experimental study of amplitude and phase detection limits in electron holography. Ultramicroscopy 64(1-4), 57-66 (1996). doi:10.1016/0304-3991(96)00019-8

49. McLeod, R.A., Bergen, M., Malac, M.: Phase measurement error in summation of electron holography series. Ultramicroscopy 141, 38-50 (2014) doi:10.1016/j.ultramic.2014.03.001

50. Cooper, D., Truche, R., Rivallin, P., Hartmann, J.-M., Laugier, F., Bertin, F., Chabli, A., Rouviere, J.-L.: Medium resolution off-axis electron holography with millivolt sensitivity. Appl Phys Lett 91 (14), 143501 (2007). doi:10.1063/1.2794006

51. Pennington, R.S., Boothroyd, C.B., Dunin-Borkowski, R.E.: Surface effects on mean inner potentials studied using density functional theory. Ultramicroscopy 159(1), 34-45 (2015). doi:10.1016/j.ultramic.2015.07.011

\section{Submit your manuscript to a SpringerOpen ${ }^{\odot}$ journal and benefit from:}

- Convenient online submission

- Rigorous peer review

- Immediate publication on acceptance

- Open access: articles freely available online

- High visibility within the field

- Retaining the copyright to your article

Submit your next manuscript at $>$ springeropen.com 